\title{
«Les chaussées sont désertes, plus de passants sur les chemins » (Esaïe 33.8)
}

La SER dans la tourmente : 1940-1945

"The highways lie waste, the wayfaringman ceased", Isaiah 33: 8. The SER in

the Storm: 1940-1945

\section{Claude Mazauric}

\section{(2) OpenEdition}

Journals

Édition électronique

URL : https://journals.openedition.org/ahrf/11078

DOI : $10.4000 /$ ahrf. 11078

ISSN : 1952-403X

Éditeur :

Armand Colin, Société des études robespierristes

Édition imprimée

Date de publication : 1 septembre 2008

Pagination : 169-207

ISBN : 978-2-200-92515-4

ISSN : 0003-4436

Référence électronique

Claude Mazauric, " «Les chaussées sont désertes, plus de passants sur les chemins » (Esaïe 33.8) », Annales historiques de la Révolution française [En ligne], 353 | juillet-septembre 2008, mis en ligne le 01 septembre 2011, consulté le 01 juillet 2021. URL : http://journals.openedition.org/ahrf/11078 ; DOI : https://doi.org/10.4000/ahrf.11078

Ce document a été généré automatiquement le 1 juillet 2021.

Tous droits réservés 


\section{« Les chaussées sont désertes, plus de passants sur les chemins » (Esaïe 33.8)}

La SER dans la tourmente : 1940-1945

"The highways lie waste, the wayfaringman ceased ", Isaiah 33: 8. The SER in

the Storm: 1940-1945

Claude Mazauric

1 Il y a deux ans, lorsque Michel Pertué et Michel Biard, puis Philippe Bourdin après eux, m'ont demandé, de préparer une communication sur la manière dont la Société des études robespierristes (SER) a passé les terribles années de la Seconde Guerre mondiale et de l'occupation, je savais que je ne disposerais pas de sources abondantes et sûres. En effet, il ne reste pas de publications « robespierristes » contemporaines des événements (ou presque pas), aucun procès-verbal de possibles réunions, ni (à notre connaissance) de papiers personnels accessibles ou de témoignages écrits des responsables d'alors de la Société, à commencer par son président, Georges Lefebvre: sur cette carence de sources directes, je reviendrai nécessairement, mais, je désirais d'entrée en abordant cette question des sources, indiquer combien la tâche qui m'était impartie se montrait périlleuse. Au surplus, il est difficile d'évoquer ces temps douloureux pour qui, alors enfant puis adolescent comme je l'étais, a vécu ce moment d'occupation militaire qui nous faisait étrangers en notre propre pays, et de surmonter le souvenir de la méfiance qui régnait alentour, des silences de la conversation entre voisins, du pas précipité des passants dans les rues (d'où la citation du prophète Esaïe qui surplombe le titre de ma communication!). Mais je ne me défilerai pas et essaierai de prendre, autant que faire se peut, la mesure exacte des choses. Naturellement, je possède quelques souvenirs de conversations avec de plus anciens que moi (Albert Soboul, Jean-René Suratteau, Marc Bouloiseau, Arthur Birembaut, Jean Dautry, Jean Bruhat, plus tardivement, Jacques Godechot, et quelques autres encore) et, à deux reprises, en 1954-1955, alors que je préparais un mémoire de DES d'histoire sur un sujet (la «dette hypothécaire paysanne » dans le Gard au cours du premier dix-neuvième siècle, sujet que Georges 
Lefebvre avait suggéré à Albert Soboul et à Camille-Ernest Labrousse) j'ai eu le privilège, désormais celui de l'âge, de rencontrer deux fois le président de la Société des études robespierristes, lequel, j'en garde le souvenir ému, a évoqué devant moi les rudes temps, les "temps de malheur ", selon son mot, de l'occupation. C'est pourquoi, malgré le risque d'échouer à présenter devant vous un papier valable, je n'ai finalement pas hésité à accepter la proposition que me faisaient les responsables de la Société et de la revue ${ }^{1}$.

2 Que savons-nous du destin de la Société au lendemain du désastre de 1940? Avec sobriété, Georges Lefebvre à la fin de 1945, en a lui-même délivré sous forme de constat, une brève esquisse: «Les années tragiques n'ont pas épargné - cela va de soi - la Société et les Annales. La Société n'a pu tenir aucune assemblée, ni renouveler son Comité; l'encaissement des cotisations s'est arrêté; le recrutement de nouveaux membres et d'abonnés est devenu impossible; en temps normal, la mort et les vicissitudes de l'existence creusaient dans nos rangs une dizaine de vides : ils n'ont pu être comblés ». Suit l'évocation de la perte de quelques membres défunts au cours des années de la guerre, et ce regret que «ce soit quelquefois par hasard, que nous nous trouvons informés de nos pertes » dont il était impossible "actuellement », disait Lefebvre fin 1945, de «faire le compte $»^{2}$. Un court rappel de la situation en 1940 nous indique que les deux premiers numéros de la revue pour 1940, réduits à « 64 pages à l'invitation du gouvernement » avaient pu paraître avant juin mais que si le numéro $3 / 4$ de juillet-décembre ( $n^{\circ} 100$ des AHRF) fut, nous dit-on, imprimé, il ne fut pas distribué ; réimprimé en février 1946 chez Georges Thomas à Nancy, ce fameux numéro a donc accompagné après la guerre la reprise de la parution régulière des AHRF : nous trouvons la preuve matérielle de cette réimpression, à la fois par la référence faite sur la quatrième de couverture du numéro, à la date du dépôt légal «II/1946 ", et à une publicité en faveur du livre de Louis Jacob, Professeur à la Faculté des Lettres de Lille, Crimes hitlériens en France. Ascq, Le Vercors, paru en 1946 dans la collection "Libération » des Éditions Mellotée à Paris ${ }^{3}$. Nous savons d'autre part qu'en octobre ou novembre 1940, après la fin de l'exode et le retour des Parisiens dans leur demeure, le ou les responsables de la Société, fut, ou furent, mis dans l'obligation de renoncer à tenir les engagements pris à l'égard des sociétaires et abonnés des AHRF auxquels ils avaient promis de distribuer le numéro 3-1940 de la revue et, une fois passée l'année 1941 que fort sagement on imaginait devoir demeurer "vacante ", on promettait aussi la reprise de « la publication régulière à compter du $1^{\mathrm{er}}$ janvier 1942, à raison de quatre numéros de 64 pages par an jusqu'à nouvel ordre ». Optimiste, malgré tout, on précisait : "Cette entreprise est nécessairement subordonnée à la rentrée des cotisations de 1940, dont très peu ont été acquittées, et de celles de 1942 à plus forte raison! " ${ }^{4}$ Il semble que le destin de la «Commission Jaurès » dont les responsabilités dans l'organisation de la commémoration du cent-cinquantième anniversaire de la Révolution en mai-juin 1939, ait connu une évolution exactement similaire, du moins si l'on suit l'étude que Christine Peyrard a consacrée à son histoire en se fondant sur le témoignage de Jacques Godechot $^{5}$. Qui d'ailleurs pouvait imaginer dans le détail ce que seraient l'occupation et la politique de Vichy? Bref, avec l'occupation et la politique de collaboration, les institutions cardinales de l'histoire de la Révolution française sont entrées dans un lourd sommeil dont elles ne sont progressivement sorties qu'au lendemain de la Libération, puis de la défaite de l'Allemagne nazie. Pouvait-il d'ailleurs en aller différemment ? Évidemment pas! 
3 La commémoration du cent-cinquantième anniversaire de 1939 avait été soigneusement préparée sous la houlette de Georges Lefebvre ${ }^{6}$. Ce dernier, Président de la Société des études robespierristes et responsable de la publication des AHRF avait été élu à cette responsabilité à la suite du décès brutal d'Albert Mathiez en 1932. L'élection de Georges Lefebvre, pour une part inattendue car il n'appartenait à aucun des cénacles politico-universitaires parisiens, signifiait en réalité la réunification dans une même cohorte de tous les historiens spécialistes de la Révolution professant des opinions républicaines, aussi bien ceux qui avaient suivi Mathiez que ceux qui n'avaient cessé de regarder avec faveur l'œuvre considérable d'Alphonse Aulard ${ }^{7}$. En 1937, Lefebvre, qui avait rendu un hommage fervent à la mémoire d'Albert Mathiez, accéda à la chaire d'histoire de la Révolution française après la retraite de son titulaire, Philippe Sagnac, lequel avait été précisément élu professeur à la Sorbonne contre Albert Mathiez en 1923, grâce au soutien d'Alphonse Aulard: l'élection de Georges Lefebvre marquait donc la fin d'une manière de schisme presque trentenaire. Pour bien souligner l'importance et l'irréversibilité du tournant opéré, Lefebvre, presque sans délai, fit créer en Sorbonne et par décret ministériel, 1'«Institut d'histoire de la Révolution française » en lieu et place du "Centre d'études » antérieur, à la direction duquel Sagnac s'était modestement illustré. En janvier 1938, Lefebvre fut officiellement adjoint à la « sous-commission ", c'est-à-dire au bureau de la «Commission Jaurès » - intitulée depuis 1960, comme le rappelle Christine Peyrard, «Commission d'histoire économique et sociale de la Révolution française » - dont il était membre depuis 1921, mais qu'il remit en mouvement à partir de 1937 et présidait en fait depuis un an déjà. Dès lors, du fait de la concentration des responsabilités, avec au centre du dispositif, la Société des études robespierristes dont le Comité directeur de trente-deux personnes comprenait dix membres qui y siégeaient déjà au temps de Mathiez et vingt-deux nouveaux élus depuis 1932, Georges Lefebvre avait tout en mains pour préparer la commémoration de 1939. Avec le soutien de ses collègues (entre autres, Marc Bloch avec qui il était en relations depuis leur compagnonnage amical et confiant à l'Université de Strasbourg8 où se trouvaient également Lucien Febvre et Maurice Halbwachs), avec C.-E. Labrousse, Émile Coornaert, Georges Bourgin, Charles Schmidt, Georges Michon, Gustave Laurent, compagnons de Mathiez, F. Braesch, Pierre Caron, anciens disciples d'Alphonse Aulard, etc., et donc de tous les membres du Comité directeur de la Société, mais aussi avec le soutien des autorités républicaines puisque la Société, nouvellement unifiée, était reconnue d'utilité publique depuis 1935, Georges Lefebvre livra la pleine mesure de son engagement d'historien et de citoyen. Il contribua à donner une ferme orientation d'histoire sociale, interrogative sur la question de la spécificité nationale du processus, éloignée de toute concession à une vision cocardière du phénomène, à l'entreprise historienne de commémoration, des cent-cinquante ans de la Révolution ${ }^{9}$. À cette fin, il prépara une fameuse "circulaire » destinée aux comités départementaux, préconisant une vaste recherche collective sur l'histoire de la bourgeoisie française, circulaire qui ne sera publiée à part qu'en 1942, on ne sait trop comment, et n'aura d'effet, comme chacun sait, qu'aux lendemains de la guerre après sa réédition et sa valorisation méthodologique. Surtout, à la demande, semble-t-il de ceux qui l'entouraient (C.-E. Labrousse, Louis Jacob, Marc Bloch), il se mit à l'écriture d'un court ouvrage destiné à un large public, ouvrage de 252 pages qui parut en mai 1939 à la Maison du Livre français, sous le titre universellement connu de Quatre-vingt-neuf ${ }^{10}$. Il y présentait la Révolution politique de 1789, dans le principal État unifié d'Europe, comme la rencontre événementielle, en un moment critique, de contradictions sociales entées sur 
des situations économiques et sur les effets de la séculaire compétition entre classes pour l'accaparement des plus-values issues de la croissance. L'auteur décelait dans l'affrontement des positions collectives, la mise en mouvement d'aspirations séculaires, venues de la longue durée, et de représentations mentales différentielles, souvent opposées, quelquefois convergentes, mais aussi l'effet de hasards divers ou encore d'initiatives de dirigeants ou de porte-parole, qui prenaient sens en raison des affrontements de classes qui, dirons-nous aujourd'hui pour faire bref, en surdéterminaient le contenu. Dans ce livre enlevé et concis, destiné à connaître après la guerre un grand succès mondial que n'a en rien compromis la médiocre riposte d'Alfred Cobban $^{11}$, Lefebvre se montrait fidèle à lui-même, entre autres à ses travaux sur la paysannerie et l'histoire agraire, non moins aux conclusions de son grand manuel de 1930, réédité en 1938, écrit en collaboration avec P. Sagnac et R. Guyot et paru dans la Collection "Halphen et Sagnac », ou aux orientations si neuves de la Grande peur de 1789, parue en 1932, et de tant d'autres écrits synthétiques. Au centre de sa démarche, une histoire sociale complexe mais fondatrice qui, rétroactivement, s'éclaire de ce que Lefebvre disait de lui-même et de sa méthode dans ce si bref essai d'égo-histoire qu'il publia en 1947 sous le titre énigmatique de "Pro Domo»: "J'ai une filiation intellectuelle : elle remonte au lycée et sans doute aussi à ma Flandre wallonne où Jules Guesde fondait le Parti ouvrier français sur la base du marxisme. Mais c'est à Jaurès que je dois le plus. C'est son Histoire de la Révolution qui a décidé de l'orientation de mes recherches $[. ..] »^{12}$. Dans son Quatre-vingt-neuf, Lefebvre décelait dès l'année première de la Révolution, le croisement interactif de multiples révolutions, "autonomes » dans leur motivation, bourgeoise, paysanne, pré-prolétarienne, etc., plaçant, tout comme Maximilien Robespierre lui-même en janvier 1792, la « révolution aristocratique » de 1787-1788 comme facteur déclenchant de la révolution politique nationale, ce qui aux yeux de Robespierre comme de Lefebvre, marquait la spécificité irréductible de la Révolution française et rendait illusoire son exportation. "Réfléchissez seulement, avait déclaré Robespierre, sur la marche naturelle des révolutions. [...] C'est ainsi que parmi vous ce sont les parlements, les nobles, le clergé, les riches, qui ont donné le branle à la révolution; ensuite le peuple a paru. Ils s'en sont repentis, ou du moins ils ont voulu arrêter la révolution lorsqu'ils ont vu que le peuple pouvait recouvrer sa souveraineté ; mais ce sont eux qui l'ont commencée ; et sans leur résistance et leurs faux calculs, la nation serait encore sous le joug du despotisme " (Discours aux Jacobins contre la guerre du 2 janvier 1792, (Euvres, tome VIII, page 83). Lefebvre, comme autrefois Jaurès, toutes différences assumées, se référait souvent, mais sans esprit de dévotion, à la pensée politique de Maximilien Robespierre.

Il était évident dans ces conditions que la Société des études robespierristes, animée par un historien qui se voulait tout à la fois, l'inspirateur et l'héritier de toute une historiographie républicaine et démocratique de la Révolution française, et qu'éclairaient en outre, depuis le début du siècle, le socialisme et le marxisme, n'avait aucune chance de trouver grâce aux yeux de l'occupant nazi et du régime de Pétain, cet "État français ", engagé, comme on sait, dès la rencontre de Montoire du 24 octobre 1940, dans la voie de la collaboration avec le Reich hitlérien, de la répression intérieure de toutes les variétés de démocratisme républicain et de la revanche sur le Front populaire. Dès l'été de 1940, les exemplaires en dépôt du Quatre-vingt-neuf de Lefebvre furent passés au pilon. Le 28 novembre suivant (peu après la date-symbole du 11), au Palais-Bourbon, devant le tout-Paris de la collaboration et des médias sous contrôle de l'époque, l'idéologue nazi Alfred Rosenberg prononçait une conférence qui fut ensuite 
publiée et diffusée sous le titre, Sang et or ou l'or vaincu par le sang. Règlement de compte avec la Révolution de $1789^{13}$. Prenant argument de l'issue bourgeoise ("ploutocratique ») de la Révolution, Rosenberg s'employait à convaincre l'auditoire que la «révolution nationale-socialiste ", en récusant les problématiques de classe au profit de l'entreprise de ségrégation et de purification raciale (le « sang »), parachevait la défaite des idées de la Révolution française et des Lumières, entachées de judaïsme, et qu'en raison de cela, le national-socialisme conduisait à la véritable émancipation des peuples européens que n'avait pu réaliser la Révolution française. L'imposition de cette idéologie fantasmagorique et raciste, opposée explicitement à l'universalisme de la Déclaration des droits de l'homme et du citoyen, visait ouvertement à séparer la nation française de ce qui, depuis cent-cinquante ans, avait contribué à construire son droit civil et le vaste corps de principes où la République, depuis 1848, puis après les années 1871-1879, avait trouvé son fondement.

5 Ainsi, la Société des études robespierristes fut-elle contrainte à se "mettre en sommeil », selon le mot de son président ${ }^{14}$, cela jusqu'en 1944-1945. Georges Lefebvre, lui-même, fit valoir ses droits à la retraite de professeur de l'enseignement supérieur à compter du 1er octobre 1941, sans possibilité de dérogation comme Vichy en accordait à d'autres plus dociles. Mais, circonspect ou bien intentionné, le Conseil de la Faculté des lettres de Paris, lui proposa de continuer ses fonctions comme «chargé de cours complémentaires ", ce qu'il fit jusqu'en mai 1945, avant son remplacement par Marcel Dunan en avril 1946. De cette période de l'occupation demeurent cependant plusieurs cours polycopiés de Georges Lefebvre, maintes fois réédités par le CDU entre 1941 et 1944 et depuis, tous portant sur l'histoire de la Révolution, «La chute du roi (1940)», «La première Terreur (1942) », « La Convention (1943) », « Le Directoire (1943-1944)»: on voit sans peine à relire ces fascicules qui se diffusaient encore lorsque j'étais étudiant en histoire entre 1952 et 1957, que Lefebvre n'avait renoncé à rien de ce qui constituait son identité intellectuelle d'homme de gauche fidèle au message de 1789 et de 1793, ni à sa raison d'être d'historien. On doit même considérer que ces "cours publics » relèvent de ce qu'on a pu appeler, peut-être assez emphatiquement au regard de ce qu'exigeait d'abnégation, la résistance politique et intellectuelle clandestine ou la résistance armée, la « résistance idéologique » à l'ordre pétainisto-nazi. Constat qui honore évidemment la mémoire de celui qui a présidé aux destinées de notre Société dans ces temps difficiles mais constat que, selon moi, nul n'avait encore souligné.

Georges Lefebvre avait à cœur dans les dernières années de sa vie de montrer son attachement aux idéaux de la Résistance et j'ai en moi le souvenir (corroboré par mon ami et ancien condisciple, Pierre Haudi-quet) de sa présence en 1954 lors d'une manifestation commémorative du 8 mai dans la cour de la Sorbonne, réunie à l'initiative de l'Union française universitaire qu'animaient des universitaires communistes, anciens résistants, comme Marcel Prenant, Jean Orcel et Guy Besse, et où il figurait, au milieu de beaucoup d'étudiants, en compagnie du doyen Châtelet de la Faculté des sciences de Paris, des philosophes Gaston Bachelard, Jean Wahl, Vladimir Jankélévitch, du linguiste Robert-Léon Wagner, des géographes, André Cholley, Pierre George, Jean Drech. Pierre Vilar, Jean Bruhat et Albert Soboul s'y trouvaient également, mais aussi beaucoup d'autres dont les noms ou les visages aujourd'hui m'échappent.

7 Y a-t-il quelques signes qui pourraient indiquer que l'activité de la Société des études robespierristes aurait pu réapparaître, ici ou là, à Paris ou, par exemple, en zone dite « libre » avant l'hiver de 1942 ? On argue à cet effet que parut en 1941, aux bons soins 
de la Librairie Nizet et Bastard, place de la Sorbonne, sous le sigle « Société des études robespierristes ", le tome II de la Correspondance de Maximilien et Augustin Robespierre, recueillie et publiée par Georges Michon, docteur ès-lettres (et vice-président de la Société en 1940) ${ }^{15}$. Devenue rarissime, quasiment introuvable, la première édition de ce recueil de 108 lettres qui complétait le premier volume paru en 1926, figure aujourd'hui en reprint dans la deuxième partie du tome III des Euvres de Maximilien Robespierre, rééditées en 2000, et de nouveau en 2007, par la Société. Nous ne savons pas dans quelles conditions a pu paraitre ce volume préparé avant la guerre. Georges Michon, lui-même, est décédé en 1945 et je n'ai pu recueillir aucun témoignage à propos de cette édition ou des conditions de sa fabrication et de son éventuelle diffusion. Au terme de la présentation de ce tome II, on lit évidemment ceci : "Nous tenons à exprimer ici toute notre reconnaissance à $\mathrm{M}$. Georges Lefebvre pour ses précieux conseils, et à $\mathrm{M}$. Paul Vaillandet pour ses utiles indications ». Nous reviendrons sur le cas de Vaillandet, devenu une figure de la collaboration avec les nazis et qui fait problème. Mais cette seule indication, à la rédaction non-datée, ne nous dit rien qui puisse laisser entendre que Georges Lefebvre aurait, même momentanément, sorti de son sommeil la Société des études robespierristes en favorisant d'une manière ou d'une autre l'édition de ce volume supplémentaire de la correspondance de Robespierre ${ }^{16}$. Aucun autre indice d'une possible activité de la Société ne nous étant apparu, nous nous en tiendrons au constat précédent de son effacement global et provisoire de 1940 à la fin de 1944. À quel moment précis cependant, la Société a-t-elle repris son activité ? Assurément, sans qu'on puisse pousser très loin l'exigence de précision, dès les lendemains de la Libération, à l'initiative de Lefebvre, lequel était en position de reprendre les contacts avec les membres de la société depuis le lieu stratégique de l'Institut d'histoire de la Révolution française où il avait continué à se rendre depuis 1940. Mais il n'y eut sans doute pas d'assemblée générale avant janvier 1945, alors que la guerre continuait, non sans difficultés inattendues pour les forces alliées occidentales en raison de la résistance de la Wermacht, en Alsace et dans les Ardennes. Mais une lettre de Georges Michon à Maurice Dommanget en date du 4 février $1945{ }^{17}$, nous apprend que l'«Assemblée des études robespierristes » s'est tenue le 21 janvier (date anniversaire de l'exécution de Louis XVI!) "sous la présidence de Lefebvre». Selon le précieux témoignage de Michon: " $Y$ assistaient P. Caron, Troux, Godechot (en uniforme d'officier d'État-major), Labrousse, Calvet [...] Aubert, Jacob, Nicolle (qui vient de publier un petit livre sur la Révolution dans la collection «Que-sais-Je?»), Javogues, deux ou trois autres que je ne connais pas, et moi ». Michon précise que «le Comité a été réélu sauf Vaillandet et de Pressac » (seule mention retrouvée par moi de ce dernier personnage qui était qualifié antérieurement, en 1940, de «conseiller municipal de Paris»). Lefebvre aurait fait savoir "qu'il démissionnerait si Vaillandet était maintenu ». On apprend aussi par cette lettre, que Georges Six renonçait à son mandat de trésorier pour des raisons de santé et qu'il «serait remplacé par Jacques Godechot après les hostilités »: en réalité, ce fut Marc Bouloiseau qui reçut la charge de la trésorerie, Godechot, celle du secrétariat adjoint plus compatible avec sa nouvelle résidence à Toulouse où il était élu professeur à la Faculté des lettres. Enfin, Michon annonçait la distribution "du dernier $\mathrm{n}^{\circ}$ de 1940 de la Revue " ("déjà composé " précise-t-il, ce qui étaie ma démonstration précédente). Ainsi, peut-on affirmer que la Société des études robespierristes s'est remise en état de marche, à peine cinq mois après la libération de Paris et de la plus grande partie du territoire national. Depuis, elle n'a jamais cessé d'exister. 
Nous avons cependant éprouvé le désir de connaître ce que sont devenus, pendant l'occupation, au-delà de Georges Lefebvre lui-même, d'autres membres de la Société, à commencer par ses responsables, les membres du Comité directeur dont la composition nous est donnée sur la page 2 du dernier numéro paru de 1940 des Annales historiques de la Révolution française. Nous en comparerons la liste à celle du même Comité directeur d'après la Libération, telle qu'elle est reproduite à la page 2 du premier numéro de 1946 ( $n^{\circ} 101$ ), en notant les retraits, car il n'y eut point d'adjonctions, et les différences d'intitulé de chacun des membres.

9 Le Président, Georges Lefebvre, comme on s'y attendait, de " professeur à la Sorbonne " est devenu " professeur honoraire à la Sorbonne ».

10 Le Secrétaire général, Louis Jacob, de « docteur-ès-lettres, professeur agrégé au lycée Janson-de-Sailly » est devenu " professeur à la Faculté des lettres de Lille » après avoir vécu la défaite de 1940 comme une insupportable humiliation, puis la période de l'Occupation en sympathie visible, au vu de ses publications de $1945{ }^{18}$, avec la Résistance. Louis Jacob était né le 28 novembre 1883 à Ugny, près de Briey, en Lorraine, à la frontière du Reich depuis le Traité de Francfort. Mobilisé au cours de la Grande Guerre, il fut envoyé au combat dans une unité de dragons, en Champagne en 1916 puis au Chemin des Dames en avril 1917, enfin dans une unité d'infirmerie : Croix de guerre avec palmes et promu sous-officier. Devenu professeur de collège dans le Nord après sa démobilisation en 1919, Louis Jacob enseigne successivement à Arras, Tourcoing, et après l'agrégation, à Lille, où il fréquente Georges Lefebvre et s'affiche comme un militant actif du Parti socialiste SFIO ; enfin il obtient en 1928 le lycée Janson-de-Sailly, à Paris, où il a désiré être muté pour y achever la thèse de doctorat qu'il préparait sous la direction d'Albert Mathiez. Il soutient brillamment ladite thèse, Joseph Le Bon (1765-1793), la Terreur à la frontière, en 1933, sous le double patronage de Philippe Sagnac et de Lefebvre. Il est dès lors intégré dans le groupe dirigeant de la Société ; on le crédite généralement d'avoir joué un rôle important dans la désignation de Georges Lefebvre à la succession d'Albert Mathiez comme Président de la SER. Le 7 avril 1934, il est d'ailleurs lui-même élu Secrétaire général de la SER et le restera jusqu'en 1946, avec le concours d'un Secrétaire général adjoint, en l'occurrence, son ami (et cadet) Jacques Godechot auquel il était très lié malgré une sensible différence d'âge, notamment en raison de leur goût commun pour l'alpinisme et sans doute en raison de leurs affinités historiographiques et politiques. Historien prolifique, passionné par l'histoire de la région du Nord qu'il aime et connaît fort bien, et par les recherches biographiques, Louis Jacob est l'auteur de nombreux articles et de plusieurs livres qui font toujours autorité, parmi lesquels se distinguent: Robespierre vu par les contemporains pour «Les classiques de la Révolution» en 1938, un Fabre d'Églantine en 1946 et le controversé Hébert, le Père Duchesne, chef des sans-culottes, paru en 1960 chez Gallimard de manière posthume. Sur le plan de sa carrière, Louis Jacob a été nommé professeur d'histoire contemporaine à la Faculté des lettres de Lille, sur " proposition du Ministère (sic) », à compter du 1er avril 1942 en remplacement de son prédécesseur, E. Braure « maintenu à la Faculté de Bordeaux ». Élu Doyen de la Faculté en février 1952, Louis Jacob se distingua comme un administrateur bâtisseur, fort apprécié par ses collègues et par les étudiants de la "Corpo.». Aux lendemains du décès de Georges Lefebvre, Jacques Godechot et Marc Bouloiseau suggérèrent le nom de Louis Jacob pour prendre sa suite à la présidence de la SER (Albert Soboul soutenant l'hypothèse de confier cette responsabilité à C.-E. Labrousse), mais son décès subit, le 1er octobre 1959, conduisit à 
une situation nouvelle qui fut conclue par la constitution d'une présidence collégiale composée de Jacques Godechot, Camille-Ernest Labrousse, Marcel Reinhard et Albert Soboul, ce dernier cumulant les fonctions de secrétaire général ${ }^{19}$.

11 Les deux vice-présidents, Henri Poulet, "conseiller d'État " ${ }^{20}$ et Georges Michon, « docteur ès-lettres », sont morts, le premier depuis le 27 mars 1941, le second, «très récemment » au moment où était composé le numéro de la revue, le 23 juillet 1945. Le trésorier, Georges Six ${ }^{21}$, « agrégé d'histoire, rue Damrémont à Paris » est remplacé par «Marc Bouloiseau, 304, Rue de Belleville » (où je l'ai connu quelques années plus tard ainsi que sa première épouse, Louise, fervente robespierriste) ${ }^{22}$; lequel, Marc Bouloiseau, est devenu chef de travaux à l'IHRF après avoir exercé les fonctions de professeur d'histoire au collège Colbert à Paris jusqu'en octobre 1945.

12 Le secrétaire général-adjoint, Jacques Godechot, « docteur-ès-lettres, professeur agrégé à l'École navale à Brest » en 1940, est devenu "professeur à la Faculté des lettres de Toulouse » depuis 1944. Jacques Godechot était d'une extrême discrétion s'agissant de lui-même ; mais j'imagine sans peine ce que fut la difficulté de vie que connurent sa famille et lui-même au cours de la guerre et de l'occupation. Mobilisé en 1939, il regagna Brest après l'armistice et sa démobilisation en août 1940, mais ne retrouva plus son poste à l'école navale où il était précédemment nommé et fut affecté au lycée de Brest. Victime des lois raciales de Vichy, il fut révoqué en décembre 1940 et gagna, avec femme et enfants, Versailles, où il trouva un emploi précaire dans l'enseignement privé, conservant le solide moral qu'on lui a toujours connu, en commençant à préparer une « histoire de l'Atlantique » dont il mit d'ailleurs à profit les données bien plus tard. La menace s'aggravant du fait du renforcement des mesures antisémites de Vichy, de l'action de la Gestapo et des rafles, les Godechot s'enfuirent de la région parisienne et réussirent à gagner Grenoble, en zone sud sous contrôle italien, en mars $1941{ }^{23}$. Ici, grâce au soutien du professeur Esmonin et à la bienveillance de la population, lui et sa famille trouvèrent un refuge, des cours complémentaires à la Faculté et quelques modestes moyens de vivre. Paradoxe: l'immeuble dans lequel ils habitaient, place Vaucanson, était aussi celui où vivait l'un des chefs de la Milice! La Résistance fit plastiquer la cage d'escalier de l'immeuble, contraignant les Godechot à s'échapper par l'escalier de service et à emménager une nouvelle fois en catastrophe ; ce fut sur le quai Créqui où ils vécurent le plus discrètement possible jusqu'à la Libération ${ }^{24}$. Jacques Godechot acheva la guerre comme officier d'État-major avant de gagner son poste de professeur à Toulouse en octobre 1945.

Parmi les vingt-sept autres membres du Comité directeur, plusieurs ont disparu: j'ai déjà évoqué le cas des deux vice-présidents; voyons celui du général de réserve Paul Joseph Diez, né en 1864 à Lunéville. Polytechnicien versé dans l'artillerie, il a rejoint le cadre de réserve depuis le 20 juillet 1918; commandeur dans l'ordre de la Légion d'honneur, connu comme un fervent républicain et un robespierriste, disciple de Mathiez, il n'a cependant pas publié une seule ligne dans les Annales révolutionnaires ni dans les AHRF de 1908 à 1940; le général Diez est décédé en 1941. Le commandant Francis Borrey, né en 1874 à Lure, ancien élève de l'École spéciale militaire de Vesoul, a fait carrière dans l'infanterie, s'illustrant notamment au cours de la Grande Guerre en 1914 puis 1915 où il fut blessé ; mais il a également servi au Maroc en 1908 puis de nouveau de 1916 à 1919, et en Chine comme conseiller militaire, d'abord en 1913-1914, puis de nouveau en 1924-1925. Officier de la Légion d'honneur, il était rayé des cadres de l'active depuis le 19 juillet 1935. Ce Franc-Comtois est l'auteur d'une thèse d'histoire 
estimable, La Franche-Comté en 1814, soutenue en 1912 mais préparée antérieurement sous la direction d'Albert Mathiez, devenu, précisément en 1911, « Professeur d'histoire et de géographie " à l'Université de Besançon. La thèse principale de Francis Borrey était accompagnée d'une thèse complémentaire consacrée à L'esprit public chez les prêtres franc-comtois pendant la crise de 1813 à 1815 dont le titre dit bien l'influence mathiéziste. En 1927 et en 1930, le commandant Borrey publia un court article dans les AHRF, «Le catéchisme de Confucius et les théophilanthropes » (1927, p. 177-178) et on fit mention dans la revue de son petit livre in- $8^{\circ}$ de 108 pages, Un sage chinois : Kou Hong Min, paru en 1930 (AHRF, 1930, p. 293). Francis Borrey a disparu au cours des années d'occupation sans que j'en sache plus sur son compte 25 .

Je n'ai trouvé aucune donnée, ni notice, relative à « Mademoiselle Duportal, docteur èslettres ", la seule femme qui était membre du Comité en 1940 et qui ne s'y trouve plus en 1946. Même carence d'information pour "De Pressac, conseiller municipal de Paris ». Autre membre du Comité directeur en 1940 qui n'est plus mentionné comme tel en 1946 : Michel Eude. Ancien élève de l'ENS, professeur agrégé d'histoire au lycée de Saint-Quentin à la veille de la guerre, il était connu pour être l'auteur de l'exposé au cours de la présentation duquel, le ${ }^{25}$ février 1932 après 15 heures, dans l'amphithéâtre Michelet de la Sorbonne, Albert Mathiez s'était effondré, victime d'une attaque cérébrale. Prisonnier en Allemagne jusqu'à la fin de 1941, Michel Eude reprit un poste d'enseignant mais perdit toute attache avec les membres de la Société jusqu'en 1945 ; par la suite, il poursuivit un temps sa carrière pédagogique dans la zone d'occupation française en Allemagne, cela jusqu'en 1952, avant de trouver un poste de chercheur au CNRS, puis de maître-assistant à l'Université de Rouen en 1966, se faisant connaître comme spécialiste de l'histoire du Comité de Sûreté générale. En 1946, soit il en déclina l'offre, soit on ne lui demanda pas de maintenir sa position au sein du Comité directeur ; mais, par la suite il se montra fidèle aux assemblées générales de la Société ${ }^{26}$.

15 Plusieurs membres du Comité de 1940 ont, en 1946, changé de situation ou de statut: Léon Dubreuil, de docteur ès-lettres est devenu Inspecteur d'académie de la Côte-d'Or, C.-E. Labrousse de « Professeur à l'École des hautes études » est devenu « Professeur à la Sorbonne » (il succédait à Marc Bloch, fusillé par les nazis, à la chaire d'histoire économique) ${ }^{27}$, Robert Schnerb, docteur ès-lettres, professeur agrégé au lycée Lakanal juste avant-guerre, est (re)devenu "professeur agrégé au lycée de Clermont-Ferrand ", après avoir vécu dans une clandestinité croissante en Auvergne où il avait trouvé refuge depuis 1941 du fait de sa révocation au titre des lois raciales de Vichy, déjà évoquées à propos de Jacques Godechot ${ }^{28}$. Le mathiéziste lorrain Albert Troux ${ }^{29}$, membre du Comité directeur depuis 1932, auteur d'une thèse remarquée sur La vie politique dans le département de la Meurthe d'août 1792 à octobre 1795 soutenue en 1936, de professeur agrégé au lycée Voltaire à Paris est devenu Inspecteur général de l'instruction publique, fonction où il fut nommé en 1941 : cette position lui valut en 1955 le refus public d'Albert Soboul, alors professeur au lycée Henri IV, d'être inspecté par «quelqu'un qui avait été nommé par le pseudo-gouvernement de Vichy qui l'avait révoqué, lui, Albert Soboul!». Pour les autres membres du Comité directeur à l'exception de ceux que je présenterai ensuite, aucune modification majeure d'intitulé n'apparaît et je ne peux pratiquement rien dire d'eux pour les années qui me préoccupent ici: cependant, je note que «François Arsac, receveur-contrôleur principal de l'enregistrement à Roubaix " devenu "honoraire ", vit désormais "en retraite à Marcillac-la-Croisille (Corrèze) ». Henri Calvet, professeur agrégé d'histoire 
au lycée Janson-de-Sailly s'y trouve encore pour peu de temps; l'ancien compagnon d'Alphonse Aulard, Pierre Caron, directeur général des Archives nationales est devenu «honoraire»; Maurice Dommanget, "diplômé d'histoire» conserve cette seule qualification ${ }^{30}$; le général Herlaut, docteur ès-lettres ${ }^{31}$, Georges Javogues, « homme de lettres", descendant du Conventionnel montagnard, Claude Javogues, fusillé par décision de la Commission militaire du Temple après l'échauffourée de Grenelle, le 10 octobre $1796{ }^{32}$, Gustave Laurent, sans qualificatif (il fut l'un des artisans de la réunification de 1924) ${ }^{33}$, Émile Le Gallo, docteur ès-lettres (onn'indique plus "professeur au lycée d'Auxerre »), Félix Ponteil, professeur à la Faculté des lettres de Strasbourg (il y est revenu), Charles-Henri Pouthas, professeur à la Sorbonne, Antoine Richard, professeur d'école normale (sans plus de précisions) ${ }^{34}$, E. Soreau, ingénieur des Arts et Manufactures, sont toujours mentionnés ; à ceux-là s'ajoutaient, en 1946 comme en 1940, les deux membres étrangers, amis de Mathiez puis de Lefebvre, Louis R. Gottschalk, professeur d'histoire à l'Université de Chicago ${ }^{35}$ et Alfred Rufer, archiviste aux Archives fédérales helvétiques, disciple de Mathiez devenu très proche de Lefebvre ${ }^{36}$.

16 Né en 1892, Georges Aubert était une figure marquante de la Société, membre de son Comité directeur depuis $1932{ }^{37}$. Ancien élève de l'ENS de Saint-Cloud, il enseigna longtemps à l'École normale de Douai et fut muté au collège Lavoisier à Paris après la suppression des écoles normales par Vichy. Vigoureux disciple de Mathiez, il s'était illustré en 1933 en protestant contre la timidité (sic!) du discours de Georges Lefebvre pour l'inauguration du buste de Robespierre à la mairie d'Arras! Mais il est le seul membre du Comité directeur de la Société dont la participation active et politique à la Résistance soit avérée : arrêté et détenu en 1943 à la prison de Fresnes, il fut, semble-til relâché, faute de preuves corroborant une dénonciation, cela, peu avant la Libération; mais je n'ai pas réussi à recueillir de plus amples renseignements sur son activité, ne pouvant de ce fait que recourir au souvenir du témoignage fiable, mais oral, des regrettés Marc Bouloiseau et Jean-René Suratteau. En octobre 1944, Aubert fut nommé au lycée Carnot où il acheva sa carrière comme censeur. Il habitait à Boulogne, à proximité de Georges Lefebvre auquel il se lia profondément dès la Libération, le visitant chaque semaine, l'épaulant, et ses disciples après lui, dans le travail préparatoire à l'édition des volumes en chantier des Archives parlementaires, cela, jusqu'à son décès en 1973 : l'assemblée générale de la Société, à la demande d'Albert Soboul et de Jacques Godechot, lui rendit alors un hommage ému et mérité.

Deux membres du Comité directeur de la Société en 1940, Paul Vaillandet, «député, professeur à l'école normale d'Avignon » en 1940 et François Vermale, "docteur èslettres ", ont connu un destin inverse de celui de Georges Aubert, celui de partisans avérés de la collaboration avec les nazis.

Né à Bessèges (Gard) en 1876 dans une famille du Vivarais de confession protestante, François Vermale avait poursuivi avec succès des études supérieures de droit et de lettres à Lyon. En 1902, il s'était installé comme avocat à Chambéry, ville où il résida jusqu'en 1930, affichant des opinions politiques avancées; il avait été candidat socialiste en 1906 à l'occasion des élections législatives, mais se montrait plutôt simplement républicain radical dans les articles qu'il rédigeait pour Le démocrate savoisien. En 1930, il entra dans la magistrature et fut nommé juge à Valence où il demeura un temps avant de gagner Grenoble. Sans doute résida-t-il professionnellement et momentanément à Valence et simultanément à Grenoble, où se 
situait son domicile familial. En effet, à partir de 1931, il figure sur la liste des habitants publiée par l'Annuaire de l'Isère: de 1931 à 1937, il y est qualifié de "sans profession ", puis de 1938 à 1943, de "professeur » sans indication d'un établissement d'affectation, l'année 1940 étant manquante. Quand devint-il à Grenoble magistrat titulaire en fonction, ou retraité de la magistrature, exerçant, à titre de remplaçant, des fonctions de juge sous la forme de suppléances ou de vacations? Les notices publiées sur lui après son décès ne le disent pas, mais si la question se pose c'est qu'on le trouve qualifié de «juge » au tribunal correctionnel de Grenoble, aux dates du 19 mars et 23 avril 1940 quand ledit tribunal fut convoqué et réuni pour juger sept militants communistes ${ }^{38}$; il en condamna plusieurs à diverses peines en application du décret du 26 septembre 1939 portant interdiction du PCF et réprimant la propagande et les activités communistes. Vermale a assisté à plusieurs réunions de l'assemblée générale des magistrats du tribunal civil de Grenoble comme juge « titulaire » et a même remplacé en juillet le juge d'instruction; mais son nom disparaît de la nomenclature judiciaire peu après sans qu'on sache pourquoi. Installé à Grenoble, François Vermale y a résidé jusqu'à son décès le 12 janvier 1970, à l'âge de quatre-vingt-quatorze ans.

Historien talentueux et érudit attentif, Vermale fut l'un des premiers chercheurs qui remarqua l'importance historique de la suppression des droits seigneuriaux en Savoie dans le dernier quart du XVIIIe siècle; il en mesura les effets et fut soucieux de les situer dans l'espace, à partir des célèbres « mappes » conservées à Chambéry. Soutenue en 1911 sous le patronage d'Albert Mathiez (qui la publia dans la Bibliothèque d'histoire révolutionnaire), sa thèse de doctorat d'État, Les classes rurales en Savoie au XVIIIe siècle, est à juste titre reconnue comme un travail de qualité qui fut, en partie, au point de départ de la grande recherche de Jean Nicolas sur l'histoire de la Savoie aux XVIIe et XVIIIe siècles. Sa vie durant, François Vermale s'est beaucoup investi dans les structures de la sociabilité savante, locale et académique: membre agrégé de l'Académie de Savoie, président de la Société savoisienne d'histoire et d'archéologie, membre de la Société archéologique de la Drôme, membre ensuite de l'Académie delphinale, il n'en négligeait ni les devoirs, ni les honneurs, se montrant dans une infinité de publications et de notes, aussi bien un spécialiste averti de l'histoire de la Révolution en Savoie et en Dauphiné (il l'abordait dans une perspective très mathiéziste) qu'analyste scrupuleux de l'œuvre de Stendhal, ou encore de celle de Joseph de Maistre vers qui l'avaient orienté ses recherches sur la franc-maçonnerie : en avril 1940, les $\operatorname{AHRF}\left(\mathrm{n}^{\circ} 2\right)$ annonçaient en placard publicitaire la sortie chez Vrin, de son édition des Considérations sur la France. Après sa mort, Vermale reçut l'hommage dithyrambique de ses pairs de l'Académie delphinale et celui des historiens de la Savoie, mais tous, cependant, observaient un silence de plomb sur son activité dans les années 1940-1944 ${ }^{39}$. J'ai relevé que sa mémoire ne reçut pas l'hommage des Annales historiques de la Révolution française auxquelles il avait pourtant beaucoup collaboré, comme historien et comme membre du Comité directeur de la Société, avant et après la Seconde Guerre mondiale. Le constatant, il m'est alors revenu qu'Albert Soboul (ou Jacques Godechot, ou Jean-René Suratteau, ma mémoire est sur ce point incertaine) m'avait indiqué qu'au lendemain de la Libération, la vigilance de Georges Lefebvre avait été surprise et qu'il avait accepté la reconduite de François Vermale au Comité directeur de la Société, parce qu'il l'estimait comme historien, mais ignorait les positions qu'il avait manifestées à Grenoble durant l'occupation. Après 1945 ou 1946, pour éviter le scandale, Lefebvre s'abstint de demander son exclusion, mais veillait, m'a-t-on dit, à ne plus le convoquer aux réunions du Comité ${ }^{40}$ ! En prévision de l'actuel 
colloque, je me suis enquis de faire le point sur cette affaire, et grâce aux suggestions et à l'aide de Jean Nicolas puis de Robert Chagny (lequel m'a transmis de précieux et démonstratifs renseignements), j'ai pu obtenir le témoignage de notre éminent collègue Paul Guichonnet, professeur honoraire à l'Université de Genève, natif de la Haute-Savoie comme moi-même, et qui fut étudiant d'histoire à Grenoble de 1941 à 1944. Son long témoignage écrit, qui est un véritable document pour l'histoire vécue de l'occupation dans les Alpes, sera publié in extenso dans les $A H R F$, avec l'accord de son auteur, et joint en document annexe à la présente communication. Mais ce qui ressort de sa lecture, est que Vermale fut bien ce propagandiste acharné à défendre le STO auprès des jeunes et à prendre fait et cause pour l'ordre nouveau hitlérien. Je ne veux ici en reproduire que les courts extraits suivants : «Je le [Vermale] revois un jour, à la bibliothèque, grand et un peu courbé, un béret sur la tête et un cache-nez autour du cou, déblatérer contre les anglo-américains et faire la propagande pour le départ des étudiants et des jeunes pour le travail en Allemagne. [...] Un autre jour, il me demanda si j'allais obéir au STO, lui ayant répondu que je n'en avais aucune intention, il s'emporta violemment et, sous les yeux effarés de l'excellent Esmonin ${ }^{41}$, me reprocha de ne pas aider l'Allemagne à gagner la guerre, etc. " Au moment de la Libération, Vermale qui s'était affiché comme un collaborationniste actif et convaincu, s'était prudemment caché dans le Massif des Bauges pendant quelques mois (combien? Nous ne le savons pas) et n'a réapparu que bien plus tard. Et Paul Guichonnet de conclure : «Je n'ai jamais su - et Esmonin, je crois, non plus - quelles avaient été les raisons qui, comme certains hommes de gauche l'avaient fait, l'ont conduit à passer au maréchalisme et à la collaboration, cause de sa mise à l'écart de la Société des études robespierristes. Ni si cette orientation pro-allemande avait été un simple délit d'opinion, ou entraîné des actes répréhensibles ".

Le cas de Paul Vaillandet est encore plus représentatif de la même dérive. Né en 1888 à Versailles, ancien normalien et ancien élève de Saint-Cloud, Vaillandet fut nommé professeur à l'École normale d'instituteurs du Vaucluse en 1924. Élève d'Albert Mathiez, robespierriste fervent, il entreprit alors de se consacrer à la préparation d'une thèse sur la Révolution dans le Vaucluse et, dans ce cadre, fut associé à la préparation de l'édition des Euvres de Robespierre, rejoignant après 1932 l'équipe que mettait en mouvement Georges Lefebvre. Historien fécond, Vaillandet a collaboré aux AHRF, y publiant quatorze articles, certains se signalant par leur évidente érudition, cinq chroniques fort pertinentes sur la Révolution dans le Vaucluse et plusieurs notices et glanes de 1926 à 1937. Membre fort actif du Parti socialiste SFIO, il devint maire-adjoint d'Avignon en 1935, et en 1936, député du Front populaire. Avec la majorité des députés socialistes, il vota les pleins pouvoirs à Pétain lors de la fameuse session parlementaire du 10 juillet 1940. Comme tous les parlementaires de la dernière Assemblée élue, il continue à percevoir son indemnité parlementaire, cela jusqu'au 31 décembre 1941. On le retrouve cependant, dès le début de 1941, réintégré dans l'enseignement et autorisé à percevoir un cumul de rémunération comme professeur au collège Arago puis au collège Colbert à Paris, établissement où enseignait également Marc Bouloiseau, lequel, regrettant l'«aveuglement " de Vaillandet pendant l'occupation, témoignera cependant en sa faveur en 1947 devant l'officier de police commis "pour supplément d'information " par la cinquième chambre civique de la Seine, saisie en appel d'une première condamnation à l'indignité nationale prononcée par contumace. Il ressort finalement de l'enquête ${ }^{42}$ que Vaillandet a suivi Marcel Déat au Rassemblement national populaire, auquel il a adhéré en 1942. Le RNP était l'une de ces grandes 
formations représentatives $\mathrm{du}$ fascisme à la française qui ont prospéré sous l'occupation avec la bénédiction de l'occupant, surtout après le retour de Pierre Laval au pouvoir. Vaillandet y exerça la fonction de secrétaire général de l'«Union de l'enseignement » qui était une organisation satellite du RNP. Comme tel il prit la parole au deuxième congrès de cette organisation en juillet 1943: discours et photo (en uniforme) de l'orateur sont reproduits dans les $n^{\circ} 56$ et 57 du National populaire. Hebdomadaire du RNP, le journal de Déat. Son nom fait aussi l'objet d'une évocation dans L'Euvre du 6 juillet 1944 ! Si Vaillandet se montrait discret sur son embrigadement devant ses collègues dans la salle des professeurs du collège Colbert, son appartenance à une organisation nationale pro-hitlérienne est avérée, et d'ailleurs par lui reconnue au cours des interrogatoires qui ont suivi. Prudent, au vu de l'évolution des choses, surtout sur le plan militaire à l'est de l'Europe, le 19 mars 1944 il avait quand même fait valoir ses droits à une pension de retraite anticipée auprès des autorités de Vichy, mais cela ne lui fut paradoxalement accordé que par la République, le 30 septembre, après la Libération! Décision évidemment retirée, au vu de son dossier et après saisine du " comité de résistance du collège Colbert », par arrêté du recteur Roussy de l'Académie de Paris, le 21 février 1945. Peu importait d'ailleurs car en août 1944, Vaillandet avait fui en Allemagne, jusqu'en Bavière, où il fut arrêté et livré par les Américains aux autorités françaises en juin 1945. Celles-ci le mirent en résidence surveillée quelque part en Allemagne jusqu'en novembre 1946, date à laquelle il revint en France pour y être jugé. Mais le temps de l'épuration était passé ! Comment et pourquoi, lui demandat-on, était-il arrivé en Allemagne? Il prétendit que ce fut contre son gré qu'il y fut entraîné; il n'aurait eu qu'un seul souci en quittant Paris le 15 août 1944 : gagner Confrancourt en Haute-Saône où il possédait une maison dans laquelle lui et sa famille pouvaient être mis à l'abri "des remous que pouvait provoquer la libération (sic)", mais le train pris à la Gare de l'Est aurait été détourné outre-Rhin par l'armée allemande sans possibilité de descendre dans les gares intermédiaires! Au bout du compte ${ }^{43}$, après jugement par contumace du 18 mai 1946 lui infligeant la dégradation nationale à vie, confiscation de ses biens à hauteur de 1000 francs et une interdiction de séjour dans les départements de la Seine, de la Seine-et-Oise et Seine-et-Marne, puis jugement contradictoire en appel le 16 juillet 1948 devant la chambre civique de la Seine, Paul Vaillandet a été finalement condamné à cinq ans de dégradation nationale, peine remise par décret du 28 mars 1949. Il a fini ses jours à Fonsorgues (HauteGaronne).

21 Comme toutes les associations et structures de sociabilité, savante ou non, la Société des études robespierristes a été confrontée au destin différent, ou aux choix divergents, de nombre de ses membres et de ses responsables, face au pétainisme et aux conséquences de l'occupation. Sans doute, pour mieux saisir tous les paramètres de l'évolution de chacun, aurait-il été convenable de remonter un peu au-delà de 1940 et de s'interroger sur ce qu'avaient pensé de la décomposition du Front populaire, de l'issue de la Guerre d'Espagne et de ses conséquences internationales, puis des accords de Munich des 29 et 30 septembre 1938 ou encore du pacte germano-soviétique du 23 août 1939, tous ces historiens, fort souvent anciens combattants de 14-18, des citoyens généralement considérés comme issus des rangs de la gauche radicale et socialiste, aucun d'entre eux, selon mes informations, n'ayant appartenant au Parti communiste, seule formation politique nationale qui ait condamné le compromis de Munich. Si, par la suite, après la guerre, Georges Lefebvre, et quelques-uns du très petit nombre de ceux qui lui étaient proches, tenaient à rappeler leur condamnation des accords de 
Munich, condamnation formulée notamment à l'occasion des manifestations du centcinquantième anniversaire de la Révolution, s'agissant des autres responsables de la Société, nous ne sommes pas en mesure d'en dire quoi que ce soit.

Observons maintenant ce qu'il est advenu des membres de la SER pendant l'occupation, au-delà du cercle dirigeant des membres du Comité directeur. Beaucoup, naturellement, ont patiemment attendu la fin de l'occupation et de la guerre. Plusieurs d'entre eux, devenus résistants de diverses obédiences, ont combattu, quelques-uns ont été victimes de la Shoah, d'autres ont subi la répression vichyssoise et nazie. C'est à ces derniers, pour autant que j'ai pu retrouver mention de quelques noms à la lecture des $A H R F$, que je voudrais consacrer la dernière partie de cette communication.

Né en 1903, Georges Simon, avocat au barreau de Reims, membre de la Société (aux assemblées générales de laquelle il était fort assidu), présidait en 1940 la section rémoise de la Ligue des droits de l'homme et assumait le secrétariat général de la Fédération marnaise des Eeuvres laïques. Tombé sous le coup de la législation antisémite et raciste de Vichy, il fut remis aux Allemands en 1941 et périt dans un camp de la mort.

Membre de la Société et chercheur, Adrien Sée travaillait assidûment, tout comme son épouse, sur l'histoire de la Révolution qui le fascinait. Il était né à Colmar en juillet 1880. Après des études de droit et de lettres à Nancy, ville-refuge de celles et ceux des Alsaciens qui demeuraient fidèles à la France, refusant de vivre dans l'Alsace annexée, il était devenu magistrat, substitut à Beauvais en 1911, procureur de la République, notamment à Paris en 1925. Tombant sous le coup de la « législation » raciale de Vichy, il fut révoqué "comme israélite" en 1940, mais aussitôt appelé par les avocats d'Orléans, où il avait exercé et où on ne l'avait pas oublié, à s'inscrire à leur barreau. Arrêté avec sa femme, le 22 (ou 23) septembre 1942, il fut transféré au camp de Compiègne et déporté à Auschwitz où ils furent assassinés dès leur arrivée. "Rendons hommage à leur mémoire. Mais le crime parle, avec des millions d'autres" écrit Georges Lefebvre en conclusion de sa notice (AHRF, 1947, p. 263).

Jean Bouvet, professeur à Mâcon aux écoles normales d'instituteurs et d'institutrices de Saône-et-Loire jusqu'en 1941, puis au collège de cette ville, était un chercheur passionné et compétent: membre de la Société, il travaillait sur la question des subsistances dans le Mâconnais, la Haute-Bourgogne et la Bresse à la fin du XVIII ${ }^{e}$ siècle et au début de la Révolution. Le 28 juin 1944, trois membres de la sinistre milice du fasciste Darnand se présentèrent à son domicile, prétendument pour une perquisition mais ils l'abattirent au milieu des siens. Au lendemain de la Libération, ses compagnons de la Résistance éditèrent en hommage à sa mémoire, le manuscrit presque achevé de son étude : La question des subsistances en Méconnais (1788-1790).

26 Jean-Marie Docq, né en 1877, était un ouvrier rémois syndicaliste, adhérent de la Société depuis 1924 et enthousiaste diffuseur dans la Marne des Annales historiques de la Révolution française. Secrétaire de l'UD réunifiée de la CGT, il était également secrétaire de la bourse du travail de Reims et présidait aussi, au titre du collège " ouvrier », une caisse nationale d'assurances sociales. En 1939, pour le cent-cinquantième anniversaire de la Révolution, il avait organisé, avec l'aide d'une jeune élève de l'École des beauxarts, Marcelle Loiseau, une grande exposition de documents dans la bourse du travail de Reims. Résistants actifs, l'une et l'autre furent dénoncées en 1944 par un gros négociant en vins ; arrêtés et déportés : on ne les revit pas. 

il était donc le cadet de quinze ans de Georges : ce fait a son importance en ce que l'aîné a fortement contribué, matériellement et intellectuellement, à l'éducation et à la formation de son frère dont il fut même, après Georges Six, le professeur d'histoire en classe terminale de philosophie, au lycée Faidherbe de Lille. Selon les témoignages de divers membres de la famille, jamais, par la suite, les liens entre les deux frères et des deux frères avec leurs sœurs et tous leurs enfants respectifs, notamment avec MarieLouise, restée seule avec quatre enfants (elle était la mère de Robert Laurent, l'auteur de la thèse bien connue sur les vignerons de la Côte-d'Or, lequel fit une honorable et longue carrière à l'Université de Montpellier) ne se sont distendus, malgré l'éloignement résidentiel des uns et des autres, notamment au moment de la débâcle de 1940. Après des études en khâgne poursuivies au lycée Lakanal, devenant plutôt géographe qu'historien stricto sensu, en raison de son admiration pour Demangeon, Théodore Lefebvre n'en fut pas moins reçu à l'agrégation d'histoire en 1914, juste avant d'être mobilisé et de servir dans une unité de mitrailleurs, en Champagne en 1915, dans la Somme en 1917, et de nouveau en Champagne où il fut grièvement blessé, cité à l'ordre de la nation, décoré de la médaille militaire. Après la guerre, il enseigna à Pau, Bordeaux, puis reçut pendant trois ans une affectation d'enseignant-chercheur en géographie à l'Université de Constantinople à l'époque d'Ataturk. Revenu en France, professeur aux lycées d'Amiens puis Charlemagne à Paris, il soutint en 1933 une thèse de doctorat d'État en géographie, écrite dans l'esprit du temps, Les modes de vie dans les Pyrénées atlantiques orientales, qui se lit encore avec intérêt. Professeur à l'Université de Poitiers, on lui confia de nombreuses responsabilités dans l'ordre de la "géographie appliquée » comme on ne disait pas encore. Mais il ne cessait pas d'être adhérent de la Société et abonné aux AHRF: mieux encore, il avait pris une part active à la commémoration à Poitiers du cent-cinquantième anniversaire. "À son initiative, m'écrit Jacques D'Hondt, le 19 juin 2007, Georges Lefebvre était venu donner une conférence au théâtre de Poitiers. Très grande affluence d'auditeurs, le proviseur avait accordé l'autorisation de sortie aux pensionnaires khâgneux, pour l'occasion; nous étions tous là. Je me souviens très bien que Madame Lefebvre (donc, la belle-sœur de Georges), avait chanté, avec beaucoup de conviction et de talent, des chants de l'époque révolutionnaire. Un peu forte me semble-t-il, elle était habillée d'une robe à rayures bleu-blanc-rouge. Tout était tricolore ! La robe faisait merveille ».

Vient alors la guerre, la défaite insupportable pour ce patriote, puis l'occupation. Je dois au philosophe Jacques D'Hondt, lui-même alors engagé dans la résistance communiste à Chinon où il débutait sa carrière de professeur, de connaître comment Théodore Lefebvre a rejoint le réseau de résistance gaulliste, constitué à partir de 1941 dans la Vienne par l'avoué Louis Renard. Ce dernier, grand mutilé de la Grande Guerre, avait réuni autour de lui dans la perspective encore lointaine d'un "débarquement " anglo-américain et de l'insurrection nationale dont l'ordre viendrait en son temps, des notables de la ville et des officiers patriotes ${ }^{45}$. Parmi ces résistants, Théodore Lefebvre et Louis Cartan, le frère du célèbre mathématicien. Jacques D'Hondt nous dit, tout à la fois, le « courage » et l'« optimisme » des conjurés du réseau Renard avec lequel il fut momentanément en relation au titre du « Front national » de ce temps, mais il évoque 
aussi la fragilité de leur camouflage, le manque de vigilance qui régnait dans l'organisation, leur candide impréparation.

Dénoncés par la police française à l'occupant, tous les membres du "réseau Renard » furent arrêtés par la Gestapo: Théodore Lefebvre le 30 septembre 1942. Transférés en Allemagne, à Wolfenbùttel, dix d'entre eux furent traduits devant un pseudo-» tribunal du peuple » nazi, condamnés et décapités à la hache le 3 décembre 1943. Le journal du Poitou, La nouvelle République a publié en date du 18 septembre 1964, la copie des originaux retrouvés en RDA, de l'ordre d'exécution et du procès-verbal d'exécution des dix martyrs, ainsi que l'annonce de la remise de la dernière lettre à leur famille de huit des condamnés, mais pas celles de Théodore Lefebvre ni de Louis Renard: nous ne pouvons connaître les raisons de ce silence épistolaire troublant venant de deux des principaux protagonistes du réseau ${ }^{46}$.

Georges Lefebvre a été profondément meurtri par la mort de son frère, survenue, en outre, deux ans à peine après la disparition de sa propre épouse, car il éprouvait pour ce frère cadet des sentiments quasiment paternels. Selon tous les témoignages de ceux qui sont entrés dans l'intimité de Georges Lefebvre, les conditions de l'exécution le laissaient sans voix: lui qui était jusqu'alors le tranquille sympathisant d'un parti socialiste modéré, un universitaire pacifiste, ouvert au compromis, peu apte à l'affichage pratique et public de ses opinions politiques, n'hésita plus alors, à plus de soixante-dix ans, à montrer son engagement. Marie-Claire Laval relève dans sa thèse quelques faits symboliquement significatifs: en novembre 1944, à propos de "l'épuration ", à ceux qui affirmaient qu'il était temps de songer au pardon pour ceux qui furent un moment égarés et qui appuyaient leur argumentation sur le fait que les Jacobins et les Sans-culottes finirent, en 1793, par ne pas sauvegarder convenablement les droits de l'accusé, Lefebvre répliquait à François Mauriac qui s'était fait leur porteparole: "Mais jusqu'à preuve contraire, aucun innocent ne périt sous nos yeux et aucun suspect n'est privé des droits de se défendre. Reste donc à rappeler cette maxime d'un fonctionnaire du Directoire: "Si la confiance dans les chefs qu'il a élus est la première vertu du républicain, la défiance est la première vertu du révolutionnaire" ». Et Marie-Claire Laval d'évoquer encore le témoignage de Richard Cobb: "Georges Lefebvre parlait généralement des leaders de la France de Vichy comme de "Messieurs les assassins de mon frère" " et Cobb rappelait "qu'il avait en outre refusé une invitation à devenir membre de l'Institut pour ne pas s'asseoir à côté des complices du meurtre de Marc Bloch. Enfin tous ceux qui ont connu l'appartement de BoulogneBillancourt ont remarqué la présence des photographies de Robespierre, de Jaurès, de Marc Bloch et de Théodore Lefebvre, son frère victime des nazis " ${ }^{47}$. Comme on sait, ce radicalisme conduisit Lefebvre à se rapprocher des communistes; on le vit par exemple lors de la journée du livre organisée par le comité national des écrivains de 1954, donner publiquement l'accolade à son compatriote, natif comme lui du Nord, Maurice Thorez, lequel était d'ailleurs un membre de la Société, abonné aux $A H R F^{48}$. Quand il me reçut à Boulogne, un jour de 1955, alors que j'achevais mon mémoire, il me déclara, tout de go: «Je connais vos opinions politiques; j'approuve votre choix». La guerre, la Résistance, la défaite de l'Allemagne hitlérienne, comme souvent, ont contribué à maximaliser les positions, à recomposer le jeu des amitiés et des relations. Georges Lefebvre ne faisait pas exception.

32 Membre de notre Société et proche d'Albert Mathiez, Alfred Chabaud avait quarantedeux ans en 1939 et tous les talents. Il avait poursuivi, en Algérie d'abord puis en 
Bourgogne et en Champagne, une carrière de professeur d'histoire mais aussi d'écrivain polygraphe qui lui avait valu une certaine notoriété : notamment, en 1937, quand il reçut un prix de l'Académie des sciences morales et politiques. Comme chercheur dans le domaine de l'histoire de la Révolution, il avait bénéficié des encouragements d'Albert Mathiez, en particulier pour un travail sur le retour de Louis XVI, passant par Épernay, après la tentative de fuite à Varennes. Il avait préparé et publié dans la collection " Les Classiques de la Révolution française " l'édition des Mémoires de Barbaroux, qu'on utilise toujours. Dès la fin de 1940, il fut, semble-t-il, sous le couvert de la clandestinité, enrôlé dans les services de renseignement de la France libre. Peu après, il rejoignit ce qui deviendra l'« Armée secrète ». À la suite de l'arrestation des officiers du génie de Reims qui avaient rallié cette formation clandestine, il fut dénoncé et arrêté par la Gestapo le 7 janvier 1944 : déporté à Buchenwald et transféré en divers camps, il est mort peu avant la défaite de l'Allemagne.

Né en 1885 à Barbezieux (Charente), voici un résistant : Robert Bizardel, membre de la Société ${ }^{49}$. Il était le neveu d'Émile Combes et sortait d'une famille de bourgeoisie moyenne anticléricale. Après des études de droit à Paris, il s'engagea dans la voie d'une carrière préfectorale qui le conduisit à la préfecture de la Lozère puis du Tarn en 1930. Résistant aux exigences de Vichy, il est rayé des cadres en 1941, rejoint son département d'origine et s'engage dans la Résistance qu'il contribue à unir et à organiser. Son action en Charente fait songer, sur un plan départemental, au grand exemple national de Jean Moulin. Il échappe de peu à la Gestapo qui l'a pris en chasse, trouve refuge clandestin à Paris et après la Libération revient en Charente (où subsiste une « poche » de résistance allemande à Royan); il y préside la commission d'épuration puis la commission militaire. Un mois après son rétablissement dans le corps des préfets, il décède, épuisé. Il collaborait fidèlement, par de solides comptes rendus, aux AHRF ainsi qu'à la Société de 1848.

Bien entendu, de nouvelles enquêtes seraient à entreprendre, des données à vérifier : puisse cette modeste esquisse en donner le désir à quelques jeunes chercheurs, plus versés que moi dans la connaissance de l'histoire de ces années terribles que j'ai certes vécues enfant puis adolescent, mais non comme historien!

Du moins, ce sera notre conclusion, éprouve-t-on le sentiment que l'immense majorité des sociétaires qui ont traversé les temps funestes de l'occupation, n'ont, ni renié leur engagement républicain en passant à l'ennemi, ni désespéré de voir renaitre la Société et sa revue. Fidèles à l'orientation que lui avaient donnée Albert Mathiez puis Georges Lefebvre, parce qu'elle s'affichait indissociablement, comme une " compagnie érudite " d'historiens de qualité, et une "société de pensée " ${ }^{50}$, fermement républicaine et démocratique, inspirée par la parole de Robespierre, nombreux furent ceux qui contribuèrent alors à sa renaissance. Rien ne l'indique mieux que la rapidité avec laquelle, dès la circulaire d'appel de novembre 1945, se sont multipliés les réabonnements aux AHRF et les formulaires d'adhésion ou de ré-adhésion : 142 réponses positives quasiment immédiates de personnes physiques furent enregistrées, parmi lesquelles, celles de 23 étrangers (dont treize venues des États-Unis). Parmi ceux-là, à côté d'anciens membres comme Marc Bouloiseau, Jacques Godechot, C.-E. Labrousse, Marcel Reinhard ${ }^{51}$, qui continuaient l'œuvre de Georges Lefebvre, bien des nouveaux venus qui assumèrent, une décennie ou deux plus tard, les principales responsabilités, au Comité directeur et à la rédaction des AHRF. 

souligner l'arrivée de trois d'entre eux, brillants historiens à venir et, déjà « anciens résistants " ne désirant pas devenir des hommes de pouvoir, et qui ont, à leur tour, marqué l'histoire de notre Société dans la seconde moitié de son centenaire: Jean Dautry, né en 1910, formé dans la tradition libertaire, devenu l'un des disciples de l'écrivain Georges Bataille et grand admirateur de son œuvre, adhérant au Parti communiste clandestin en 1942, un historien d'un gigantesque savoir, infatigable résistant qui fit le coup de feu pour la Libération de Paris. Hélas ! il est trop tôt disparu, en 1968. Albert Soboul, évidemment ${ }^{52}$, né en 1914 , révoqué par Vichy pour avoir contribué à l'organisation de la manifestation patriotique du Front national, le 14 juillet 1942 à Montpellier, et qui fut intégré dans l'hiver 1943-1944 dans le réseau de résistance du Musée des Arts et traditions populaires, animé (entre autres) par son ami Charles Parain. Jean-René Suratteau, enfin, né en 1916, ancien khâgneux du lycée Louisle-Grand où il côtoya Albert Soboul, son ainé de deux ans, à la Fédération des étudiants révolutionnaires. Ancien socialiste proche de la gauche "pivertiste » dont il s'était éloigné en 1939-1940, Suratteau s'affilia dès 1943 au réseau «Ceux de la Résistance (CDLR) », où il rencontra de futurs hiérarques de la République gaullienne : Bourdeau de Fontenay, futur créateur de l'École nationale d'administration, Léo Hamon ${ }^{53}$, futur ministre, Michel Debré, futur concepteur de la Constitution de la Ve République, etc. Clandestin agissant sous un faux nom depuis l'automne 43, Suratteau fut élu membre du conseil exécutif de CDLR en mars 1944 et, à la demande du Conseil national de la Résistance, gagna immédiatement la ville de Laval, dans le département de la Mayenne, pour y préparer avec succès l'action des Forces françaises de l'intérieur au moment du futur débarquement allié. À la Libération, il fut nommé secrétaire général de la préfecture de Seine-et-Marne, puis, tout simplement, en décembre 1944, démissionnant de toutes ces responsabilités politiques ou administratives qui risquaient de l'entraîner où il ne voulait pas aller, c'est-à-dire, au-delà de ce qu'avait exigé le combat libérateur, il se mit à la disposition de Georges Lefebvre à l'IHRF, recruté à la Sorbonne comme « agent technique du CNRS ». Dès lors, après avoir été admis sans coup férir au premier concours normal de l'agrégation, en 1945, commença pour lui une belle carrière de chercheur et d'historien universitaire qu'il acheva comme doyen de la Faculté des lettres et des sciences humaines de l'Université de Bourgogne à Dijon ${ }^{54}$.

Ils étaient un peu mes maîtres, mais surtout mes amis et des inspirateurs : c'est en évoquant leur mémoire, eux qui aujourd'hui ne sont plus, que j'ai voulu achever ce parcours dans l'histoire de notre commune Société des études robespierristes, arrivée au terme de ses cent ans d'existence et à la veille d'un nouveau départ, qui, j'en forme le vœu, ne la montrera pas infidèle à ce qui demeure, en permanence, sa raison d'être : l'histoire et la démocratie dans toute l'extension que porte son concept.

\section{ANNEXES}




\title{
Témoignage de Paul Guichonnet, Professeur honoraire à l'Université de Genève « à Claude Mazauric »
}

\author{
Annemasse, le 28 avril 2007
}

Mon cher collègue, Je vous envoie :

- Des éléments sur la biographie de Vermale,

- Mon témoignage personnel sur son attitude pendant le gouvernement de Vichy ».

[Ici des tirés-à-part et photocopies de notices nécrologiques, en plus de quelques considérations personnelles et indication de correspondants potentiels].

« Lorsque Vermale quitta la Savoie - où il conserve des attaches - pour Valence, puis Grenoble, il est toujours un homme de la gauche avancée et franc-maçon. Les deux départements savoyards vivent alors à l'ère du radicalisme et le socialisme est un courant encore minoritaire.

Sur Vermale à Grenoble.

Il doit y arriver après sa retraite de magistrat, vers 1936 et il s'insère dans le milieu des historiens et des universitaires, où il était déjà connu, par le voisinage de Chambéry et par ses travaux historiques.

La période 1940-1945 est, à Grenoble, particulièrement difficile, avec les restrictions et la pénurie, aggravées par le repli de nombreuses gens, venues de la zone occupée - dont de nombreux Juifs, qui seront rapidement inquiétés et arrêtés.

La ville, qui n'avait pas oublié sa tradition révolutionnaire de 1789, était, avant la guerre, un fief radical-socialiste. À 19 ans, après un an d'hypokhâgne au lycée du Parc à Lyon, classe transférée à Clermont-Ferrand, j'y arrive en 1941, nanti d'une bourse de licence et y fais la licence d'histoire et géographie, puis le diplôme d'études supérieures et quelques suppléances au lycée Champollion. Grenoble ressent, évidemment, le traumatisme de la défaite et passe sous le régime de Vichy, qui exerce sa propagande de la Révolution nationale, qui comme ailleurs, dérivera vers la Milice et la collaboration [au lycée, c'est le lever des couleurs et le chant de « Maréchal, nous voilà !»].

Le 2 février 1943 a été créé le Service du travail obligatoire en Allemagne, auquel je suis astreint. Je refuse de répondre aux convocations, ce qui, à Grenoble, devient difficile pour les « réfractaires ", à cause des vérifications de la police, puis de la Milice et, à la fin de cette année, je retourne me mettre à l'abri dans ma Savoie, où les gendarmes étant acquis à la Résistance, déclarent à la police que les « requis » sont absents ou introuvables.

À Grenoble, l'opinion est partagée et les étudiants sont l'objet de la propagande de Pétain. Elle s'exerce - comme pour tous les Français - par la presse, les affiches, la radio et les actualités du cinéma et, spécialement, par des conférences à l'Association des étudiants. C'est là que le « bel Armand »Caraccio, italianisant et futur professeur à l'Université, dont la femme est directrice du lycée de jeunes filles, exalte la Révolution nationale et que le dernier fils, posthume, de Péguy, présente son père comme un « fasciste français ». L’Université est partagée. Les professeurs y sont alors écoutés et respectés. Le recteur Marcel Blanchard, grand mutilé de 14-18, tient un mémorable discours « maréchaliste », à l'ouverture de l'année universitaire de 1942 et il est sifflé 
par les étudiants, ce qui provoque chez lui une colère homérique. Le doyen de la Faculté des lettres est le philosophe Jacques Chevalier qui, bien que dégommé du Ministère de l'Éducation nationale de Vichy exalte Pétain avec une adulation grotesque : « Le maréchal sait tout ! » et déblatère contre les francs-maçons, les instituteurs laïques, les socialo-communistes du Front populaire, qui ont conduit la France à la ruine. Certains professeurs vont plus loin, comme Gallay, enseignant le droit romain et l'histoire ancienne, qui sera exécuté par des résistants (Grenoble est alors une université de format modeste : pas de Faculté de pharmacie ; études de médecine dont le cycle se termine à Lyon ; pas de chaire d'histoire ancienne, enseignée par le professeur de droit romain. Ce qui fait dire au caustique Raoul Blanchard, beau-frère du recteur Marcel et portant le même patronyme que ce dernier, « Grenoble est la seule université française qui ne jouit pas de toutes ses facultés »!).

Il y a aussi les gens hostiles à Vichy. Ils le sont surtout par le dépit de la défaite de 1940 et l'animosité, non pas tant au nazisme, que contre les Allemands et les Italiens. (Je me rappelle encore, après la chute de Mussolini et l'occupation de la zone sud-est par les Allemands, la joie des Grenoblois (j'y étais !) bien insouciants et ignorant de l'avenir ! regardant défiler la garnison italienne, désarmée et conduite vers l'internement par les soldats d'Hitler). Le sentiment anti-allemand était présent dès 1938-1939, avec les discours de Daladier, et les deux millions de prisonniers étaient la préoccupation majeure, après la défaite. La collaboration de Pétain avec Hitler, commencée à Montoire, suscitera ensuite une haine croissante, mettant dans le même sac, Laval, la Milice et Hitler, à cause des exactions dans la lutte contre la Résistance. Mais les camps de concentration, vaguement connus, passaient après les camps de prisonniers de guerre et c'est après 1945 que nous aurons le choc d'apprendre l'extermination des juifs et des déportés.

Les étudiants en histoire et géographie avaient deux professeurs chefs de file, le géographe Raoul Blanchard et l'historien Edmond Esmonin. Blanchard était la personnalité dominante, ami orléanais de Péguy, grand bourgeois, dreyfusard, ayant le cœur à gauche et le portefeuille à droite! Comme plusieurs générations de ses élèves, j'ai été très marqué par ce grand mandarin à l'ancienne mode, très introduit dans l'establishment politique et militaire et les milieux industriels de la houille blanche. Par l'orgueil, le côté imperator, il avait quelque chose d'un Braudel avant la lettre, comme je le constaterai par la suite. Blanchard, qui avait créé la géographie au Canada français, était un libéral à l'anglaise, qui se trouvera très à l'aise avec les Américains, après leur arrivée, et il sera très ami avec Yves Farge, commissaire de la République à Lyon. Respecté - et redouté - il n'avait aucunement sacrifié au pétainisme, dont il se tenait soigneusement à l'écart, se moquant des «bondieuseries cléricalo-maréchalistes » de Jacques Chevalier et s'irritant des discours pompeux et tricolores de son beau-frère Marcel. Ami d'André Siegfried, alors que tout le monde de Vichy déblatérait contre la République, il m'avait fait faire un diplôme d'études supérieures de géographie électorale, sur « la géographie et le tempérament politique dans les montagnes de la Haute-Savoie ", publié en 1943 dans la Revue de géographie alpine (première étude sur ce thème, après le Tableau politique de la France de l'Ouest de Siegfried (1913), dans un genre qui deviendra la tarte à la crème de la sociologie électorale, après 1945-1950).

L'historien Edmond Esmonin était d'un tout autre genre. Ce $18^{\mathrm{e}}$ miste, dernier élève de Lavisse, bourguignon de Gevrey-Chambertin, ami de Roupnel, était le prototype du radical-socialiste de la « Troisième », très lié à Herriot, peut-être franc-maçon et 
nettement anti-clérical. Il m'avait pris en affection et je lui rendais souvent visite. Il était, bien qu'ayant bravement combattu en 14-18, profondément et viscéralement hostile à Pétain, pour des raisons idéologiques de vieux démocrate républicain. Il était l'ami des anti-Vichy grenoblois, comme le doyen des sciences, Gosse, qui sera abattu par les Allemands. J'étais, comme on dit maintenant, sur la même longueur d'onde que lui : des parents instituteurs publics (mon père a fabriqué des générations d'instituteurs à l'école annexe de l'École normale de la Haute-Savoie) et petit-fils d'un « instituteur Jules Ferry » président de la Société républicaine de Passy! Donc de sensibilité des " hussards noirs de la République », dans une idéologie radicale socialiste, au sein de laquelle j'ai fait mes premières armes de conseiller municipal, en 1953, avant que ce parti ne disparaisse de la scène politique. Esmonin était un homme de courage tranquille. Il soutenait moralement le malheureux Louis Halphen, nommé à Grenoble, le seul juif qui, avec Marc Bloch, n'avait pas été chassé de la Sorbonne. J'ai compris, plus tard, la détresse du ménage Halphen, dont la bibliothèque et les papiers avaient été volés par les Allemands, dans leur bel appartement de Paris. Ce frêle professeur et sa menue épouse étaient complètement désemparés, vivant dans une chambre spartiate de la modeste pension d'étudiants, où moi-même habitais. Je leur rendais quelques petits services, comme faire la queue pour retirer leurs cartes d'alimentation. Dans leur chambre : le lit, un lavabo à l'eau froide, fort peu de chauffage, une petite table et une seule chaise, Madame Halphen s'asseyant sur leur petite malle de bois... Esmonin accueillait aussi Jacques Godechot, que j'ai beaucoup vu plus tard en Italie, où il s'occupait de ses jacobins. Godechot, chassé comme juif de son poste de professeur (je crois à l'École navale de Brest), se cachait à Grenoble. Dans une petite salle du département d'histoire, il nous donnait, sous l'autorité d'Esmonin, à un petit groupe d'étudiants « sûrs » des exposés sur la Révolution et le Directoire.

Après ces longues digressions, qui vous ont peut-être paru fastidieuses et que je vous prie d'excuser, mais que justifie peut-être le souvenir très présent que j'ai gardé de cette époque et de ces événements, j'en viens maintenant à François Vermale. Il était installé à Grenoble, âgé de 65-66 ans en 1942-1943 et, bien que cet âge passât alors pour celui d'une vieillesse largement avancée, il était alors très vert et plein de pugnacité.

Vermale et Esmonin étaient très amis, pour deux raisons. Tout d'abord les affinités politiques de gauche et ensuite l'histoire « républicaine » et en particulier celle de la Révolution, dont le souvenir était toujours très vivant à Grenoble (avec la nuance Aulard-Danton chez Esmonin et Mathiez-Robespierre chez Vermale). Esmonin était, à l'ancienne formule, un historien de la politique et des institutions, dont la référence était la Revue historique, auteur d'une thèse sur « la taille en Normandie sous Colbert " (dont Blanchard, qui le détestait, se gaussait : «Comment peut-on passer dix ans de sa vie à étudier une telle connerie!»). Curieusement, c'est Blanchard qui m'a fait connaître les Annales, auxquelles, comme tous ceux de ma génération, j'ai beaucoup sacrifié, avec, dans cette ère pré-Braudel, l'histoire rurale de Marc Bloch et les fondements géographiques de l'histoire de Lucien Febvre.

À un moment - 1942-1943 - où, dans le milieu universitaire et intellectuel de Grenoble, le pétainisme - fût-il délirant comme celui d'un Jacques Chevalier -était encore le discours dominant, Vermale était un « collaborationniste » notoire, avec une fixation particulière pour l'exécution du STO. Je le revois un jour, à la bibliothèque, grand et un peu courbé, un béret sur la tête et un cache-nez autour du cou, déblatérer contre les Anglo-américains et faire la propagande pour le départ des étudiants et des jeunes pour 
le travail en Allemagne. Autre souvenir : j'avais fait la connaissance de Vermale chez M. Esmonin et, un autre jour où nous étions tous les trois dans son bureau, il me demanda si j'allais obéir au STO. Lui ayant répondu que je n'en avais aucune intention, il s'emporta violemment et, sous les yeux effarés de l'excellent Esmonin, me reprocha de ne pas aider l'Allemagne à gagner la guerre, ajoutant ces paroles que, 64 ans plus tard, je puis encore répéter textuellement : « Si vous vous dérobez au devoir du STO, le gouvernement du Maréchal va prendre contre vous une mesure terrible : la mort civile. Vous n'aurez plus d'existence légale ; vous n'aurez plus d'identité, vous ne pourrez pas vous marier, faire un testament, vous serez rayé de la société ! ».

Quelque temps plus tard, Ed. Esmonin me dit qu'il avait « définitivement rompu une amitié de trente ans avec ce malheureux Vermale».

J'entendis dire, par la suite, qu'après la Libération, Vermale s'était caché -peut-être dans une résidence secondaire - dans le massif des Bauges, au-dessus du lac d'Annecy et qu'il avait échappé, de justesse, à des résistants, venus pour l'abattre. Vermale allait encore vivre pendant un quart de siècle mais, tout au moins en Savoie, il disparut du petit milieu des historiens et des sociétés savantes, sans publier d'études, sa grande période ayant été celle des années 1910-1935. Je ne le rencontrai plus, et pour les gens de ma génération, son nom n'était plus qu'une référence bibliographique. Je n'ai jamais su - et Esmonin, je crois, non plus -quelles avaient été les raisons qui, comme certains hommes de gauche l'avaient fait, l'avait conduit à passer au maréchalisme et à la collaboration, cause de sa mise à l'écart de la Société des études robespierristes. Ni si cette orientation pro-allemande avait été un simple délit d'opinion, ou entraîné des actes répréhensibles.

Bien que voisins des Dauphinois, nous ne sommes pas trop au courant des recherches de leurs historiens. Si Vermale a été inquiété après la Libération, cela a dû laisser des traces dans l'histoire de cette période de Vichy et de la Résistance, qui a certainement été très étudiée par les historiens et chercheurs des deux universités grenobloises. Je vous conseille de vous adresser, pour avoir, là-dessus, des renseignements et des références, à notre collègue Pierre Bolle, d'une de ces universités, dont vous trouverez l'adresse, bon connaisseur de cette période. Ou encore à la très active et compétente Hélène Viallet, Savoyarde, qui a été en poste à Annecy, actuelle directrice des Archives départementales de l'Isère (2, boulevard des Adieux, 38000 Grenoble).

Voici ce que je puis vous dire sur Vermale, sans doute trop longuement. Cela m'a fait revivre une époque et un milieu dont je suis un des rares survivants. N'hésitez pas à m'écrire, si je puis encore vous être utile dans votre recherche et acceptez l'expression de mes sentiments très amicalement et attentivement dévoués.

Paul Guichonnet

\section{NOTES}

1. Je tiens à remercier pour leur aide, les renseignements qu'ils ont pu me communiquer, les pistes qu'ils m'ont suggéré d'explorer, Jean Bart, Jean-Paul Bertaud, Roger Bourderon, Robert Chagny, Jean Charles, Jean-François Condette, Janine Cholvy, Marc Cholvy, Jean-Pierre Jessenne, Jacques D'Hondt, Raymond Huard, Marie-Claire Laval, Jean Nicolas. J'exprime mon infinie gratitude à Anne Simonin, spécialiste éminente de la France sous l'Occupation, pour son aide 
précieuse et désintéressée, et à mon compatriote savoyard, Paul Guichonnet, professeur honoraire à l'Université de Genève, pour son inestimable témoignage écrit, lequel sera placé en pièce annexe à la suite du présent texte.

2. Annales historiques de la Révolution française (AHRF), 1946, $\mathrm{n}^{\circ} 101$, page 1.

3. Dans le long article d'hommage qu'il consacre à la vie et à la carrière de Louis Jacob - nous y reviendrons - Louis Trénard écrit ceci à propos de cette publication: "La deuxième guerre mondiale détermine une coupure dans l'œuvre de Louis Jacob. Les combats de la Résistance lui suggèrent en 1946 un récit historique Ascq et le Vercors [...] La documentation sur le massacre d'Ascq a été curieusement saisie en 1944 lors de la retraite allemande : le camion qui renfermait les rapports des responsables a été accidenté en Tiérache ». En avril 1944, quatre-vingt-six personnes de la commune d'Ascq avaient été massacrées par les SS sans qu'il ait été possible aux assassins de formuler le moindre prétexte pour tenter de se "justifier" : il semble que même la presse vichyssoise l'ait constaté ! Louis TRÉNARD, "Louis Jacob et la région du Nord », AHRF, $\mathrm{n}^{\circ} 160,2-1960$, p. 147.

4. AHRF, $1940, \mathrm{n}^{\circ} 100$, p. 1.

5. Ch. PeyraRd et M. Vovelle (dir.), Héritages de la Révolution française à la lumière de Jaurès, Aix-enProvence, PUP, 2002, p. 46-47. En complément de cet ouvrage, pour une plus grande précision, on doit consulter dans les $\operatorname{AHRF}\left(\mathrm{n}^{\circ} 108,1947, \mathrm{n}^{\circ} 4\right.$ p. 73) le compte rendu de «L'assemblée générale de la Commission centrale et des Comités départementaux d'histoire économique de la Révolution » (1939), t. 1 et 2, 1942 (soulignépar moi) et 1945 par Georges Lefebvre.

6. Sur Georges Lefebvre, on se reportera à la belle thèse de doctorat en science politique, de Marie-Claire LAVAL (sa petite-nièce), soutenue devant la Faculté de droit et des sciences économiques de Dijon, le 10 mars 1978, préparée sous la direction de Claude Courvoisier: L'historien et le peuple. Un exemple: Georges Lefebvre, 2 vol. Les aspects biographiques sont à rechercher au tome 1, p. 17-59. On se reportera également aux recherches de Stéphane Buzzi dont l'article «Georges Lefebvre (1874-1959), ou une histoire possible », Le Mouvement social, $\mathrm{n}^{\circ} 200$, 2002, p. 177-195, montre la proximité de l'historien avec les fondateurs des Annales, Marc Bloch et Lucien Febvre, ses collègues de l'Université de Strasbourg dans les années 1930, en plus de son intérêt majeur pour la sociologie et de ce que nous désignons aujourd'hui par «l'histoire des mentalités». Voir aussi le numéro d'hommage des AHRF n 159, janv.-mars 1960 et l'article classique d'Albert Sовоul sur «l'œuvre de Georges Lefebvre » a paru dans le n 3 de 1975 des AHRF pour le centième anniversaire de la naissance de l'historien. Il existe des notes de lecture de Georges Lefebvre et des papiers de recherches à l'IHRF (Université de Paris 1) mais divers papiers personnels que son neveu Robert Laurent, fils âné de sa sœur Marie-Louise-1 (1884-1957), avait conservés, ont disparu en 1997 dans l'incendie du chalet que ce dernier possédait à Malbuisson (près de Pontarlier) dans le Jura. D'autres papiers, repris après le décès de Georges Lefebvre par l'une de ses deux filles (il s'agit de Marthe, la cadette, professeur d'histoire, car Marie-Louise-2, cadre hospitalière à Paris, est décédée antérieurement à lui), retrouvés par le frère de MarieClaire Laval (que je remercie de son aide) donnent quelques pistes informatives sur les liens et les relations familiales de Georges Lefebvre avec ses proches.

7. Ici, se pose la question, maintes fois soulevée, de l'appartenance politique, avérée ou supposée, des membres du Comité directeur de la SER, d'abord tel qu'il se configura à la suite de la réunification de 1924, puis après l'élection de Lefebvre en 1932, enfin jusqu'à nos jours. Si l'on retient comme indicateur de l'orientation politique dominante de la SER à la veille de la guerre, la composition de son Comité directeur en 1940, on remarque que la totalité des responsables de la SER adhéraient ou étaient proches, toutes nuances doctrinales effacées, du Parti radical et radical-socialiste et du Parti socialiste SFIO. Aucun n'appartenait au Parti communiste français, quoique plusieurs, socialistes de nuance plutôt guesdiste comme C.-E. Labrousse, ou anciens disciples d'Albert Mathiez, aient manifesté, comme Mathiez lui-même, de la sympathie pour la Révolution russe de 1917 avant de se détourner du communisme dans les années 1920-1930. Seul, 
en 1940, se distinguait au milieu de cet environnement politique d'une gauche plutôt modérée (mais pas modérément républicaine !), l'instituteur Maurice Dommanget, venu de la "gauche » de la Fédération unitaire de l'enseignement et qu'on savait (ou disait) proche de la mouvance trotskyste, quoique dans la vie intérieure de la SER il ne soit jamais sorti de l'horizon stratégique et théorique, défini successivement par Mathiez puis par Lefebvre (cf. note 30, ci-après). Nombre de ceux qui composaient le Comité directeur étaient réputés franc-maçons. Aux lendemains de la Libération, la situation ne connut d'infléchissement sensible qu'en raison de l'inclination nouvelle manifestée par Georges Lefebvre en faveur du Parti communiste, mais sans que cela n'entraînât la moindre modification dans la composition politique ou l'orientation d'ensemble du Comité directeur. Dans la liste des 142 personnes abonnées aux AHRF en 1946, auxquelles la circulaire de 1945 demandait quelles étaient celles qui adhéraient simultanément à la SER, six ou sept seulement de ceux qui répondirent positivement, adhéraient alors, selon mes investigations, au Parti communiste français : outre Maurice Thorez, il s'agissait de Jean Bruhat, Jean Dautry, Albert Soboul, Michel Hugonnot, Roger Garmy; peut-être faudrait-il ajouter un ou deux patronymes à cette courte série, mais il est évident que la SER ne devint « communiste » que dans les fantasmes mac-carthystes des chevaliers de l'« ordre occidental »! En 1951, au vu de la liste des abonnés, aucun changement notable n'est perceptible. Après 1960, malgré l'arrivée en position de responsabilités importantes au sein de la SER d'historiens connus par ailleurs comme des adhérents du PCF, tels qu'Albert Soboul, François Hincker (qui s'éloigna du parti en 1980), Michel Vovelle, tout au cours de la séquence bicentenariale, ou de moi-même in fine, jamais la Société des études robespierristes, et moins encore son Comité directeur, ne sont devenus des succursales politiques du communisme français, comme les courants qui lui étaient hostiles le laissaient entendre (cf. ci-après, p. 8 et 9).

8. Malgré son départ pour la Sorbonne, je note ici les liens conservés par Georges Lefebvre avec l'histoire de Strasbourg et de l'Alsace, notamment en raison de sa proximité avec Jean-Henri Poulet et Paul Leuilliot ( 1897-1987), auteur d'une belle monographie d'histoire révolutionnaire (Les Jacobins de Colmar, 1923), bientôt Secrétaire de rédaction des Annales fondées par Lucien Febvre et Marc Bloch, futur directeur de recherches à la sixième section de l'EPHE, et auteur d'une monumentale histoire de l'Alsace en trois volumes.

9. « Georges Lefebvre " par Marc BOULOISEAU, dans Historical Dictionnary of the French Revolution, ed. by S. F. ScotT and B. Rothaus, Greenwood Press, Conn., 1985, t. 2.

10. Propriété de l'IHRF, Quatre-Vingt-Neuf a été réédité chez Armand Colin en 1950, puis par Albert Soboul aux Éditions sociales en 1970, Quatre-vingt-neuf a été traduit en anglais dès 1947 par l'éminent professeur R.R. Palmer et édité à Princeton University Press (seconde édition en 1949) sous le titre The Coming of the French Revolution, en italien par le disciple de Lefebvre, le très influent historien Alessandro Galante Garrone (L'Ottantanove) et édité par Einaudi à Turin en 1949, et, à la suite, dans de nombreuses langues; la dernière édition connue est inscrite au catalogue de Deutscher Tagenbuch Verlag, à München depuis 1989, sous le titre 1 ?S9, Das Jahr des Revolution, avec une préface de Claude Mazauric.

11. The Social Interpretation of the French Revolution, London, 1964.

12. AHRF, $1947, \mathrm{n}^{\circ} 2$, pages $188-190$.

13. Le contenu de la diatribe de Rosenberg suscita une riposte argumentée presque immédiate, en deux textes successifs : "L'obscurantisme au XXe siècle » et « Révolution et contre-révolution au XX ${ }^{\mathrm{e}}$ siècle ", rédigés par le philosophe Georges POLITZER depuis sa résidence clandestine, avec l'accord de la direction clandestine du PCF ; ce fut la seule riposte à notre connaissance, à la provocation de Rosenberg; elle fut publiée en février-mars 1941 en 96 pages dans une livraison de La pensée libre, reprise clandestine, de la revue de Paul Langevin, La Pensée, réactivée par Jacques Decour, Jacques Solomon et Georges Politzer lui-même, éditée par le Parti communiste français sous la responsabilité de Jean Jérome, son responsable éditorial, et diffusée aux universitaires de la région parisienne dont le nom figurait sur le Livret de l'étudiant de 1939. Roger 
Bourderon et moi-même en avons réédité le texte en 1984 aux Éditions sociales avec une fine et neuve présentation par Roger BOURDERON, sous le titre Politzer contre le nazisme. Écrits clandestins (février 1941). Georges Politzer fut peu après arrêté et fusillé par l'occupant le 23 mai 1942 au Mont Valérien, en compagnie de Félix Cadras, Arthur Dalidet, André Pican, Georges Dudach et le docteur Bauër. Selon son ami et condisciple, l'Inspecteur général Henri DuBIEF ( Un homme : Jean Dautry », AHRF, 1968, p. 427), c'est la lecture de cette riposte qui détermina Jean Dautry, jusqu'alors plutôt proche de l'ultra-gauche, de Georges Bataille et du surréalisme, à adhérer au Parti communiste français clandestin.

14. Marc Bouloiseau, en 1962, reprit à son compte cette expression lors d'une réunion du Comité départemental d'histoire de la Révolution de la Seine-maritime, présidée par Jean Vidalenc, et à laquelle j'étais présent.

15. Né le 6 octobre 1882 à Corménon (Loir-et-Cher) dans une famille bourgeoise d'entrepreneurs de Travaux publics, Georges Michon poursuivit de brillantes études à l'école alsacienne puis au collège Sainte-Barbe. Étudiant d'Aulard, puis par ses études devenu grand connaisseur du droit maritime britannique et international, il devint docteur en droit en 1913; il fit ensuite carrière comme cadre dans des compagnies maritimes, y compris, au service de la République de 1914 à 1918. Se rapproche de Mathiez après 1919 et soutient en 1924 une belle thèse qui demeure précieuse : Essai sur le Parti feuillant: Adrien Duport (Payot éd.) qui lui vaut le doctorat ès-lettres ; il publie ensuite la fameuse Correspondance inédite de Barnave. Vivant visiblement de ses rentes pour l'essentiel ou de travaux occasionnels, on le vit simplement « chargé de cours » en divers lycées parisiens, mais s'il est surtout connu pour sa publication de la « Correspondance de Maximilien et Augustin Robespierre " dans les Euvres de Maximilien Robespierre, déjà évoquées, on ne saurait oublier le reste de ses écrits qui se sont succédés à un rythme étourdissant : 1928 : Documents pontificaux sur la démocratie et la société moderne; 1931: Clemenceau ; 1935 : La préparation de la guerre. La loi de trois ans ; 1937 : Robespierre et la guerre révolutionnaire; 1941 : Le rôle de la presse en 1791-1792. Il avait rendu hommage à la mémoire de Mathiez dans deux articles très importants : "Albert Mathiez, rénovateur de l'histoire de la Révolution » dans la revue de Jean-Richard Bloch, Europe, et "Albert Mathiez, journaliste» dans le Bulletin d'histoire révolutionnaire, de la "Commission Jaurès ». Gustave Laurent, son ami, nous dit de lui : «Il avait, comme Albert Mathiez, conservé intacte la foi profonde des républicains en la tradition robespierriste d'où découlèrent au siècle suivant, les véritables principes d'une démocratie basée sur les conceptions constructives d'une société mieux organisée et véritablement populaire ». Qui dit mieux ? (Cf. AHRF, 1947, p. 264-267.)

16. Dans son adresse «Aux membres de la Société des études robespierristes et aux abonnés des Annales », parue dans le numéro 101 de la revue (janv.-mars 1946) et qui marquait la reprise de sa parution, Georges Lefebvre précisait ceci : "Pendant l'occupation, M. G. Michon a publié luimême (soulignépar nous) un Supplément à la Correspondance de Robespierre. On peut se le procurer à la Librairie Nizet et Bastard »: on ne saurait mieux dire que la Société n'était pour rien dans cette édition! La question demeure : comment Michon a-t-il fait?

17. Cette lettre ( $\left.{ }^{\circ} 538\right)$ est conservée aux Archives nationales dans le Fonds Maurice Dommanget (Carton 14/AS/333). Je remercie Julien Louvrier de me l'avoir fait connaître. Il semble en outre au travers de cette lettre de Michon, que ce dernier qui était encore chargé de fonctions «à la Recherche scientifique » où il risquait de "n'être pas maintenu [...]» ayant déjà été menacé d'être «limogé en 1941 », car le "successeur de Lefebvre n'aurait peut-être pas les mêmes intentions que lui sur l'Institut de la Révolution » : effectivement, ce successeur qui devait être Marcel Dunan, un historien de la Bavière à l'époque napoléonienne, laissa péricliter l'Institut jusqu'à l'arrivée de Marcel Reinhard en 1956. Georges Michon décéda peu après.

18. Cf. note 3 .

19. Je remercie Jean-Pierre Jessenne pour son aide, Jean-François Condette, maître de conférences à l'IUFM du Nord-Pas-de-Calais, pour m'avoir communiqué copie des documents qui 
ont rendu possible l'énoncé des précisions ci-dessus. Je remercie aussi Martine Aubry du centre de recherches historiques de Lille 3, dont la diligence dans la transmission des sources a fait merveille. Sur Louis Jacob, on pourra consulter pour en savoir plus : le numéro spécial déjà évoqué à la note 3 des AHRF d'avril-juin 1960 (articles de Jacques Godechot et de Louis Trénard, successeur de Louis Jacob à l'Université de Lille), le numéro de la Revue du Nord (1954, tome 36) publié en hommage à Louis Jacob (articles du Recteur Michel Souriau et de Roger Portal). Les Annales de l'Université de Lille (années 1941-1942, 1944-1945, 1951-1952, 1953-1954), témoignent de l'activité de Louis Jacob, dans et pour sa faculté. La livraison 1941-1942 nous apprend que «l'interruption du contrôle de la circulation à la frontière de la «zone interdite » a permis au plus grand nombre de revenir à la faculté au mois de janvier (1942)... Mais parmi eux, ce ne fut pas le cas des philosophes Vladimir Jankelevitch et Henri Gouhier, chassés de leur chaire en application de la «législation » raciale du pseudo-gouvernement de Vichy. Note incidente : on apprend par la livraison de 1944-1945 des Annales, que «M. Jankelevitch qui, pendant l'occupation allemande, avait dû quitter notre Faculté, a été réintégré dans ses fonctions ; par un arrêté ministériel du 29 mai 1945, il a été nommé professeur titulaire de Philosophie morale et Science de l'éducation, à compter du 1er mars 1942 (souligné par nous) » : c'est à des faits de ce genre qu'on mesure ce que fut véritablement la «Libération »... Mais comment omettre la responsabilité des autorités de Vichy qui secondèrent si bien les desiderata de l'occupant, dans la « zone interdite ", mais aussi ailleurs!

20. Né à Thiaucourt en Meurthe-et-Moselle, la même année (1874) que Mathiez et Lefebvre, JeanHenri Poulet était fils de magistrat ; il a poursuivi les habituelles brillantes études des jeunes de son milieu, au lycée Louis-le-Grand, puis aux facultés de droit et de lettres de Paris, avant de devenir Secrétaire particulier à la Présidence de la République sous Émile Loubet, en 1906. Maître des requêtes au Conseil d'État, il a rejoint le Front puis est devenu préfet du Haut-Rhin dans l'Alsace reconquise en 1918. Retraité en 1920 à l'âge de 46 ans, il s'est dès lors consacré à l'histoire érudite de la Révolution, multipliant les comptes rendus dans les Annales révolutionnaires puis dans les AHRF On lui doit quelques belles monographies bien connues : Saint-Mihiel en 1792, L'esprit public à Thann pendant la Révolution, etc. Jusqu'à son décès en 1941, Poulet fut un fidèle de Mathiez puis de Lefebvre.

21. Né à Lille le 18 décembre 1870, Georges Six devint professeur agrégé d'histoire, exerçant notamment au lycée Faidherbe où il connut et se lia à Georges Lefebvre, et enseigna son frère cadet, Théodore. Mobilisé, capitaine dans la territoriale, il a combattu en Champagne, puis sur le front de Macédoine, jusqu'en 1918 (Croix de guerre, Légion d'honneur). Un temps Inspecteur d'Académie, il revint à l'enseignement, notamment en classe préparatoire au lycée Condorcet. Retraité en 1933, il se consacra à l'achèvement de son grand œuvre, toujours indispensable : Dictionnaire biographique des généraux et amiraux de la Révolution et de l'Empire, 1934, couronné en 1937 par l'Académie française. Trésorier de la Société de 1932 à 19...45 ! Son dernier article fut la notice nécrologique qu'il écrivit à la mémoire de son élève, Théodore Lefebvre (voir ci-après note 41). La Société rendit un vibrant hommage à sa mémoire après son décès le 26 février 1947.

22. Notice nécrologique par Albert SoBoul, AHRF, 1974, p. 56.

23. On lira, à ce propos, aux fins de comparaison, l'exceptionnel et très nouveau récit-témoignage de Jean-Pierre KAMINKER, linguiste à l'Université de Perpignan, La persécution contrariée: les Kaminker à Valréas (1943-1944) entre antisémitisme d'État et bienveillance d'une population, Limoges, Lambert-Lucas éd., 2007, 492 pages.

24. Pour ce récit de ce que fut la vie de Jacques Godechot de 1940 à 1944, je me fonde sur plusieurs conversations que j'eus avec lui entre 1983 et 1988 quand le l'ai assidûment fréquenté au CTHS (Comité des travaux historiques et scientifiques), données corroborées par le témoignage de son fils, Monsieur Thierry Godechot, par les souvenirs de son élève, mon collègue Claude Petitfrère, Professeur émérite à l'Université de Tours, par le témoignage de Paul Guichonnet, évoqué ci-après, et en m'appuyant sur le mémoire de «Lauréa » de Livia RizonETTI, 
soutenu en 2001 à l'Université La Sapienza de Rome, mémoire préparé sous la direction de mon collègue Carlo Ghisalberti, Professeur d'histoire du droit dans cette université, membre de l'Istituto per la Storia del Risorgimento et dont le titre (traduit) est celui-ci : « Jacques Godechot : un profil bio-bibliographique ». Sur l'œuvre de Jacques Godechot, on se référera, en premier lieu, aux notices nécrologiques et hommages de Michel VovelLe, AHRF, 1989, p. 169-170 et de Claude PetiTfRère, AHRF, 1990, p. 308-317.

25. Je remercie le colonel F. Guelton, Chef du département de l'Armée de terre, de m'avoir communiqué copie des États de service du général Diez et du commandant Borrey, conservés au Service historique de la défense à Vincennes. À propos du commandant Borrey, j'ai reçu du service de l'état-civil de la ville d'Amance en Haute-Saône, copie de son acte de naissance, le 18 juillet 1874 à Lure, sous le nom de Francis Arquinet, légitimé sous le nom de Borrey après le mariage à Vesoul le 14 février 1875, de sa mère Françoise-Joséphine avec François Borrey, "propriétaire », et copie de son propre mariage à Amance, le 25 juin 1903, avec Julie-Adèle Ecoffet; mais ni l'état-civil de Lure, ni celui d'Amance, n'ont pu m'indiquer la date, ni le lieu de son décès pendant la Seconde guerre mondiale, données qui ne leur ont pas été communiquées ou n'ont pas été enregistrées. L'état-civil de Lunéville qui m'a communiqué copie de l'acte de naissance de Paul Joseph Diez, le 16 octobre 1864, indiquant qu'il était fils d'un professeur au lycée de Sens et d'une sage-femme de Lunéville, n'a pas été plus en mesure de me renseigner sur la date, ni le lieu, du décès de celui qui est devenu le général Diez. Sur ces deux personnages, le Service historique de la défense n'est pas en position de faire connaître ce qu'il est advenu d'eux après qu'ils ont été rayés des cadres d'active et de réserve de l'armée de terre.

26. Notice nécrologique par J.-R. SURATTEAU, AHRF, 1989, p. 75. Je précise, pour la petite histoire, que Michel Eude et moi, avons enseigné en même temps, peu avant et peu après Mai 1968, l'histoire de la Révolution aux étudiants de l'Université de Rouen, sous la libérale direction de Jean Vidalenc, mais sans doute pas en l'abordant de manière identique ! Sur Michel Eude, on se reportera au travail de Clyde Plumauzylle, jeune historienne de l'Université de Paris 1, qui présente une communication au présent colloque.

27. Traiter ici de l'œuvre et de l'action de Camille-Ernest Labrousse au sein de la SER, et plus généralement de son influence dans le champ de l'histoire de la Révolution française, dépasserait notre propos, s'agissant de la période qui fixe notre attention : les années 1940-1945. Si Georges Lefebvre ne cesse de louer la grande portée heuristique des travaux de Labrousse pour l'intelligence du processus révolutionnaire, cela depuis 1937, il faut aussi noter que l'auteur de l'histoire du mouvement des prix, des salaires et des revenus depuis 1730 puis de la crise conjoncturelle de 1789, écrit peu dans les AHRF, que son charisme de directeur de recherches s'affirme postérieurement à 1945 et qu'il n'exerce de responsabilités réelles dans la SER qu'après la mort de Lefebvre, se contentant jusqu'à sa participation à la «présidence collégiale » mise en place en 1960, de soutenir de son éloquence l'action du Président Lefebvre et de ses principaux disciples à la direction de la Société et des AHRF. Pour approcher l'œuvre et l'action de Labrousse au regard des questions évoquées dans cet article, on se reportera au numéro spécial des AHRF, consacré à la mémoire de C.-E. Labrousse, n²76, 1989 ; cf., en particulier, les articles de Michel VovelLE, p. 99-107 et de Guy LEMARCHAND, p. 132-143.

28. Notice nécrologique , AHRF, 1963, p. 129.

29. Notice nécrologique par Jean-René SURATTEAU, AHRF, 1981, p. 507.

30. Sur Maurice Dommanget, son œuvre importante d'historien de la Révolution et sa " posture » de " pacifiste » avant et au cours de la Seconde guerre mondiale, je renvoie au colloque qui s'est tenu à Beauvais à sa mémoire en 1994 (Maurice Dommanget. Actes du colloque international tenu à Beauvais les 6 et 7 mai 1994, Beauvais, 1995, Archives départementales de l'Oise et Conseil général de l'Oise, éditeurs) et à la communication présentée dans le présent colloque par mon collègue et ami Serge Bianchi. Dommanget a traversé la période 1940-1945 dans un attentisme prudent. 
31. Richard Cobb a rendu hommage à la mémoire d'Auguste-Philippe Herlaut, "Général républicain ", spécialiste fort savant du fonctionnement de la direction des opérations et des bureaux de la guerre en l'an II de la Première République, quand s'y trouvaient à l'œuvre nombre de militants sans-culottes, cela dans un bel article : AHRF, 1965, p. 502.

32. Sur Georges Javogues et, parmi d'autres, aux articles qu'il avait consacrés à son ancêtre et parus de 1922 à 1928, on se reportera à la nécrologie parue dans les AHRF, en 1963, p. 123.

33. Il y aurait beaucoup à dire sur le rôle majeur de Gustave Laurent dans l'histoire de la Société et de sa revue. Né en 1891 dans une famille de moyenne bourgeoisie, une véritable dynastie de républicains engagés, tant du côté paternel que maternel depuis la Révolution elle-même, élève brillant au lycée Janson-de-Sailly, on le vit, avec le vieux communard Lissagaray, aux premiers rangs des manifestations contre le Thermidor de Victorien Sardou en janvier 1891, manifestations qui conduisirent Clemenceau à déclarer : «Il faut qu'on le sache, la Révolution est un bloc dont on ne peut rien distraire ». Le jeune Laurent, élève puis étudiant d'Aulard, a renoncé jeune à une carrière lucrative de négociant en Champagne pour se faire historien, occupant d'abord un poste de greffier au tribunal de première instance de Reims - ville où il s'est d'ailleurs fixé pour la vie de manière à mieux se rapprocher des archives ! De 1906 jusqu'à son décès le 9 novembre 1949, il a publié un grand nombre d'ouvrages savants, toujours utilisés par tous les historiens. Notons : Les Cahiers de doléances des communautés d'habitants de l'ancienne généralité de Châlonspour les États généraux de 1789 (4 vol.1906-1930), en 1933, une Histoire de Reims, en 1939, l'édition dans le cadre des Euvres de Maximilien Robespierre, de ses journaux : Le Défenseur de la Constitution, préparant aussi l'édition des Lettres à ses Commettants parue après sa mort. Conseiller municipal de Reims et souvent adjoint au maire de 1900 à 1940, inscrit à gauche, il fut débarqué de sa position par Vichy; on lui doit l'existence depuis 1921 de la première voie publique urbaine dédiée à Robespierre, le Boulevard Robespierre. En 1924, il favorisa le rapprochement entre les Annales révolutionnaires de Mathiez, de qui il était devenu proche, avec la Revue historique de la Révolution française que Charles Vellay avait créée après sa brouille avec Mathiez et dont il avait repris la direction. Comme chacun sait, ce rapprochement donna naissance aux Annales historiques de la Révolution française dont il fut le co-directeur jusqu'en 1931. Il soutint vigoureusement Georges Lefebvre après son élection à la présidence de la Société en 1932. La Société lui doit beaucoup. Il existe quelque part (où ? je l'ignore...) un fonds Laurent comportant des centaines de lettres et de notes de lecture que personne encore n'a exploité. Pour en savoir plus, on se reportera, entre autres, à la nécrologie signée d'Édouard VeLLAY, parue dans les AHRF en 1950 (n 118, p. 184-191).

34. Notice nécrologique dans AHRF, 1948, p. 192.

35. Louis Gottschalk (1899-1971) a présidé l'American Historical Association. Historien de La Fayette et l'Amérique, il avait débuté par une biographie de Marat et fréquenté Mathiez dans les années 1920. Membre du Comité directeur de la Société de 1934 (sur la suggestion de Lefebvre), il le resta solidement jusqu'à sa mort. Cf. la belle notice de Robert PALMER (AHRF, 1976, p. 665-668).

36. Érudit impressionnant de savoir et historien de la République helvétique qu'il admirait, autant que de Pestalozzi dont il montrait l'originale conception de l'éducation, Alfred Rufer fut le maître de J.-R. Suratteau (cf. AHRF, 1987, p. 235 sq).

37. Notice nécrologique par Marc BOULOISEAU, AHRF, 1974, p. 155.

38. Leurs noms et qualités : Henri Turrel, 29 ans secrétaire régional du PCF, déjà emprisonné depuis septembre 1939, Line Balte, directrice d'école maternelle, Paul Nouvel, chef d'équipe aux PTT, secrétaire du Secours populaire français, Marius Molens, employé des chemins de fer, trésorier du Secours populaire français, Georges Roche, Martial Roux, Hyppolite Liaud, tous trois, infirmiers à l'hôpital psychiatrique de Saint-Egrève, arrêtés et jugés à la suite d'une perquisition faite sur « dénonciation ». Je dois toutes ces informations à quelques découvertes occasionnelles faites dans les archives judiciaires par mon collègue et ami Robert Chagny que je remercie.

39. Bulletin de l'Académie Delphinale, 8e série, 9e année, $\mathrm{n}^{\circ} 4$, avril 1970, sous la signature de Claude Boncompain (pharmacien et stendhalien réputé que j'ai connu et fréquenté à Valence dans la 
Drôme, lorsque j'y fus nommé professeur au lycée Émile Loubet, du 1er octobre 1957 jusqu'au 1er octobre 1959), Gilbert DALLET et Jean OHERNE. L'histoire en Savoie, $\mathrm{n}^{\circ} 101$, mars 1991, cf. «Petit dictionnaire des historiens de la Savoie ", notice de E. Compain, p. 117.

40. On est cependant surpris de trouver dans le $n^{\circ} 117$ (1950), à la page 62-63, une lettre de «M. Vermade" (lapsus calami qui en ferait dire long au Dr Freud!) dans laquelle Vermale, toujours bon historien, s'employait, non sans efficacité, à rectifier une appréciation de Gustave Laurent sur la position sociale de Jean-Baptiste Drouet, le maître de poste de Varennes... Mais comme Laurent venait de mourir, la discussion demeura sans suite.

41. Alors professeur d'histoire moderne à la Faculté des Lettres de Grenoble.

42. Je remercie Anne Simonin, chargée de recherches au CNRS en poste à Oxford, sans laquelle, je n'aurais pas disposé de tous les moyens de disposer des informations dont ce développement peut faire état et qui sont extraites du dossier Z5/136 dossier 6030 des Archives nationales. Je remercie la Direction des Archives nationales de m'avoir autorisé, à ma demande, à consulter ce dossier qui ne sera librement communicable qu'en 2058.

43. De l'étude minutieuse des pièces du dossier Vaillandet conservé aux Archives nationales, il apparaît que ce dernier a quitté son appartement de cinq pièces, sis au 44 boulevard Beaumarchais à Paris, le 15 août 1944, en compagnie de son fils, lui-même milicien, avec « deux grosses valises", des "fourrures" et une "voiture d'enfant», selon le témoignage de la concierge de l'immeuble, témoignage recueilli par le commissaire de police Raoul Farge du commissariat de la Roquette (rapport du 15 mars 1945). On signale aussi que beaucoup de visiteurs fréquentaient l'appartement des Vaillandet, semble-t-il, à la sollicitation du fils ; mais parmi eux pas d'allemands, du moins en uniforme. D'autre part, en juillet 1944 - donc après le débarquement allié en Normandie, mais avant l'offensive décisive- Vaillandet tenait encore des réunions publiques pour le RNP du «chef » Marcel Déat: le journal L'Euvre nous annonce la tenue de deux réunions avec Vaillandet comme orateur, la première, le 6 juillet, 7, Rue Dupuytren pour la 6e section du RNP, le 7 juillet pour la 11e section, au 5 avenue Parmentier. La perquisition légale faite au domicile de Vaillandet par le commissaire Farge, n'a livré que de rares objets épars : parmi eux des périodiques (le magazine nazi Signal, des exemplaires du National populaire), un recueil des allocutions radiodiffusées de Philippe Henriot en 1944.

44. On se reportera à la notice nécrologique rédigée par Georges Six dans "In Memo-riam ", AHRF, $\mathrm{n}^{\circ} 101,1946$, p. 68-70.

45. On lira l'admirable récit de Jacques D’H ONDT, «Un enseignant de philosophie sous l'occupation allemande (1941-1943) ", Revue philosophique, n 3/2002, p. 261 à 292.

46. L'ordre d'exécution est daté du 16 novembre 1943, sous le sigle du "Reichsminister der Justie » et le procès-verbal d'exécution sous celui du "Uberstaatsumwalt bei dem Landgericht » du 10 décembre. Les geôliers allemands contraignirent par la force au silence, les condamnés quand ils entonnèrent La Marseillaise au moment de mourir.

47. Marie-Claire Laval, thèse citée, I, p. 57-58.

48. Cf. la liste des 142 "Membres et abonnés » (parmi lesquels 119 français) constituée à la suite des réponses à la "Circulaire de novembre 1945 ", publiée dans le nº 103 de 1946 des AHRF Maurice Thorez y est désigné comme « vice-président du Conseil, Rue de Varennes ».

49. AHRF, «In Memoriam », 1947, n 101, p. 66.

50. C'est ainsi que, fort justement, le regretté François Hincker, alors Secrétaire général de la Société avait en 1993 qualifié la Société des études robespierristes.

51. Il faut ici rappeler le rôle important, et sous-estimé généralement, joué par Marcel Reinhard, successeur de Marcel Dunan à la Sorbonne et prédécesseur d'Albert Soboul, dans le développement de la SER après la mort de Lefebvre : lui qui aurait souhaité que la Société cessât de cultiver son attache robespierriste et mathiéziste qu'il jugeait politiquement contreproductive après 1958, revint sur son jugement et demeura fidèlement membre de la présidence collégiale, avec Godechot et Labrousse, Soboul en étant Secrétaire général, jusqu'à son décès en 
1973. Je lui dois personnellement beaucoup, tant pour les encouragements qu'il me prodigua que pour son soutien lors de la polémique avec François FURET qui suivit la parution de ma chronique relative au livre que ce dernier avait publié, avec Denis RICHET, en 1966-1967, sur l'histoire de la Révolution. Historien majeur du Directoire (Le Département de la Sarthe sous le Directoire, 1935), Reinhard est l'auteur de deux grands livres, Le Grand Carnot (2 vol. , 1950-1952) et La Chute de la royauté (1969) ; il fut l'introducteur de la préoccupation démographique dans l'histoire sociale et militaire de la Révolution. On se reportera à l'hommage des AHRF, sous la plume d'Albert SoBouL et de J.-R. SuRATTEAu (1974, p. 1-14.)

52. Cf. mon essai :Un historien en son temps : Albert Soboul (1974-1982). Essai de biographie intellectuelle et morale, suivi des entretiens d'Albert Soboul des 4 mars et 5 août 1981 avec Raymond Huard et MarieJosèphe Naudin, Nérac, Éd. d'Albret, 2004, 251 pages.

53. Jean-René Suratteau retrouva Léo Hamon, alors ministre de la République, en tant que membre du jury de la thèse de doctorat en science politique, de Marie-Claire Laval sur Georges Lefebvre, le 10 novembre 1978 à l'Université de Dijon ; autres membres du jury, outre le directeur de la recherche, C. Courvoisier, Jean Bart et J. Delaunay.

54. On se reportera au numéro 316, avr.-juin 1999, des AHRF consacré, en partie, à évoquer la mémoire de J.-R. Suratteau, décédé en octobre 1998 à Vence.

\section{RÉSUMÉS}

Malgré l'extrême pénurie de sources directes émanant de la Société des études robespierristes, l'auteur s'attache à reconstruire l'histoire de cette société d'historiens pendant l'occupation de la France, depuis l'été de 1940 jusqu'aux lendemains de la Libération, et même jusqu'à la reprise d'activité de la SER en janvier 1945, et de la publication des Annales historiques de la Révolution française au début de 1946. Mettant à profit, souvenirs vérifiés et témoignages, données issues des archives ou encore des notices nécrologiques des principaux animateurs et adhérents de la SER, l'auteur suit le destin individuel et collectif de la plupart des historiens français, spécialistes de l'époque révolutionnaire, en activité entre 1930 et 1960. Il montre le rôle considérable qui fut celui de Georges Lefebvre dans cette histoire et dans la résurrection de la SER en 1944-1945, mais évoque aussi le profil, l'action, l'opinion et les choix politiques de plusieurs dizaines d'autres, tous également confrontés aux sollicitations contraires qui caractérisaient ces années, les pires dans l'histoire contemporaine de la France.

Despite the paucity of direct sources from the Société des études robespierristes itself, the author aims to reconstruct the history of this organization of historians during the period of the Occupation, from the summer of 1940 until the days following the Liberation, and even until the renewal of professional activity of the SER in January 1945, and the publication of the Annales historiques de la Révolution française at the beginning of 1946. Relying on personal accounts and eye-witness reports, material from archives, even obituaries ofthe principal leaders and adherents of the SER, the author follows the individual and collective destinies of most of these French historians, specialists of the Revolutionary period, active between 1930 and 1960 . He shows the considerable role played by Georges Lefebvre in this history and in the revival ofthe SER in 1944-1945. And he evokes the profiles, actions, opinions and political choices of scores of others, all confronted by the political choices during these years, years that rank as the worst in the contemporary history of France. 
INDEX

Mots-clés : collaboration, histoire sociale, nazisme, occupation, résistance, sociabilité scientifique, historiographie, Georges Lefebvre

\section{AUTEUR}

\section{CLAUDE MAZAURIC}

(Ancien membre de la présidence collégiale de la SER)

Université de Rouen 17, Rue Notre Dame 30000 Nîmes claude-simone.mazauric@wanadoo.fr 